\title{
One-Dimensional Behavior of Dilute, Trapped Bose Gases
}

\author{
Elliott H. Lieb ${ }^{1, *}$, Robert Seiringer ${ }^{1,2, \dagger}$, and Jakob Yngvason ${ }^{2}$ \\ 1. Department of Physics, Jadwin Hall, Princeton University, \\ P. O. Box 708, Princeton, New Jersey 08544 \\ 2. Institut für Theoretische Physik, Universität Wien, \\ Boltzmanngasse 5, A-1090 Vienna, Austria
}

August 14, 2003

\begin{abstract}
Recent experimental and theoretical work has shown that there are conditions in which a trapped, low-density Bose gas behaves like the one-dimensional delta-function Bose gas solved years ago by Lieb and Liniger. This is an intrinsically quantum-mechanical phenomenon because it is not necessary to have a trap width that is the size of an atom - as might have been supposed - but it suffices merely to have a trap width such that the energy gap for motion in the transverse direction is large compared to the energy associated with the motion along the trap. Up to now the theoretical arguments have been based on variational - perturbative ideas or numerical investigations. In contrast, this paper gives a rigorous proof of the one-dimensional behavior as far as the ground state energy and particle density are concerned. There are four parameters involved: the particle number, $N$, transverse and longitudinal dimensions of the trap, $r$ and $L$, and the scattering length $a$ of the interaction potential. Our main result is that if $r / L \rightarrow 0$ and $N \rightarrow \infty$ the ground state energy and density can be obtained by minimizing a one-dimensional density functional involving the Lieb-Liniger energy density with coupling constant $\sim a / r^{2}$.

This density functional simplifies in various limiting cases and we identify five asymptotic parameter regions altogether. Three of these, corresponding to the weak coupling regime, can also be obtained as limits of a three-dimensional Gross-Pitaevskii theory. We also show that Bose-Einstein condensation in the ground state persists in a part of this regime. In the strong coupling regime the longitudinal motion of the particles is strongly correlated. The Gross-Pitaevskii description is not valid in this regime and new mathematical methods come into play.
\end{abstract}

\footnotetext{
${ }^{*}$ Work partially supported by U.S. National Science Foundation grant PHY 01-39984.

${ }^{\dagger}$ Erwin Schrödinger Fellow, supported by the Austrian Science Fund.

(C) 2003 by the authors. This paper may be reproduced, in its entirety, for non-commercial purposes.
} 


\section{Introduction}

The technique of trapping and cooling atoms, that led to the first realization of Bose-Einstein condensation (BEC) in dilute alkali gases in 1995 [5, 18], has recently opened the possibility for experimental studies, in highly elongated traps, of Bose gases that are effectively one-dimensional. Some of the remarkable properties of ultracold one-dimensional Bose systems with delta function interactions, analyzed long ago $[22,23]$, may thus become accessible to experimental scrutiny in the not too distant future. Among these are pseudo-fermionic behavior [12], the absence of BEC in a dilute limit $[21,36,14,34,10]$, and an excitation spectrum different from that predicted by Bogoliubov's theory [23, 17, 20]. The paper [33] by Olshanii triggered a number of theoretical investigations on the transitions from $3 \mathrm{D}$ to an effective 1D behavior with its peculiar properties, see, e.g., [6, 7, 8, 11, 13, 19, 32, 35, 39]; systems showing the first evidence of such a transition have recently been prepared experimentally $[4,15,16,38]$.

Until now the theoretical work on the dimensional cross-over in elongated traps has either been based on variational calculations, starting from a three-dimensional delta-potential [7, 13, 33], or on numerical Monte Carlo studies [1, 3] with more realistic, genuine 3D potentials but particle numbers limited to the order of 100 . This work is important and has led to valuable insights, in particular about different parameter regions $[8,35,32]$, but a more thorough theoretical understanding is clearly desirable since this is not a simple problem. In fact, it is evident that for a potential with a hard core the true $3 \mathrm{D}$ wave function does not approximately factorize in the longitudinal and transverse variables and the effective one-dimensional potential can not be obtained by simply integrating out the transverse variables of the $3 \mathrm{D}$ potential. In this sense the problem is more complicated than in a somewhat analogous situation of atoms in extremely strong magnetic fields $[2,30]$, where the Coulomb interaction behaves like an effective one-dimensional delta potential when the magnetic field shrinks the cyclotron radius of the electrons to zero. In that case the delta potential can be obtained formally by integrating out the variables transverse to the field in a suitable scaled Coulomb potential. With a hard core, on the other hand, where the energy is essentially kinetic, this method will not work since it would immediately introduce impenetrable barriers in 1D. The one-dimensional effective interaction emerges only if the kinetic part of the Hamiltonian and the potential are considered together.

In the present paper we start with an arbitrary, repulsive 3D pair potential of finite range and prove rigorously that in a well defined limit the ground state energy and particle density of the system are described exactly by a one-dimensional model with delta-function interaction. This is a highly quantum-mechanical phenomenon with no classical counterpart, since a $1 \mathrm{D}$ description is possible even though the transverse trap dimension is much larger than the range of the atomic forces. It suffices that the energy gap associated with the transverse confinement is much larger than the internal energy per particle.

While the three-dimensional density remains low (in the sense that distance between particles is large compared to the three-dimensional scattering length) the 
one-dimensional density can either be high or low. We remark that, in contrast to three-dimensional gases, high density in one dimension corresponds to weak interactions and vice versa [22]. In this paper we shall always be concerned with large particle number, $N$, which is appropriate for the consideration of actual experiments. In order to make precise statements we shall typically take the limit $N \rightarrow \infty$ but the reader can confidently apply these limiting statement to finite numbers like $N=100$.

Besides $N$, the parameters of the problem are the scattering length, $a$, of the two-body interaction potential, and two lengths, $r$ and $L$, describing the transverse and the longitudinal extension of the trap potential, respectively. To keep the introductory discussion simple let us first think of the case that the particles are confined in a box with dimensions $r$ and $L$. The three-dimensional particle density is then $\rho^{3 \mathrm{D}}=N /\left(r^{2} L\right)$ and the one-dimensional density $\rho^{1 \mathrm{D}}=N / L$. The case of quadratic or more general trapping potentials will be considered later. We begin by describing the division of the space of parameters into two basic regions. This decomposition will eventually be refined into five regions, but for the moment let us concentrate on the basic dichotomy.

In earlier work $[27,26]$ we have proved that the three-dimensional GrossPitaevskii formula for the energy (including its limiting 'Thomas-Fermi' case) is correct to leading order in situations in which $a$ is small and $N$ is large. This energy has two parts: The energy necessary to confine the particles in the trap, which is roughly $\left(\hbar^{2} / 2 m\right) N\left(r^{-2}+L^{-2}\right)$, plus the internal energy of interaction, which is $\left(\hbar^{2} / 2 m\right) N 4 \pi a \rho^{3 \mathrm{D}}$. The trouble is that while this formula is correct for a fixed confining potential in the limit $N \rightarrow \infty$ with $a^{3} \rho^{3 \mathrm{D}} \rightarrow 0$, it does not hold uniformly if $r / L$ gets small as $N$ gets large. In other words, new physics can come into play as $r / L \rightarrow 0$ and it turns out that this depends on the ratio of $a / r^{2}$ to $\rho^{1 \mathrm{D}}=N / L$. As we shall show, the two basic regimes to consider in highly elongated traps, i.e., when $r \ll L$, are

- The one-dimensional limit of the three-dimensional Gross-Pitaevskii/'ThomasFermi' regime

- The 'true' one-dimensional regime.

The former is characterized by $a L / r^{2} N \rightarrow 0$, while in the latter regime $a L / r^{2} N$ is of the order one or even tends to infinity (which is referred to as the Girardeau-Tonks ${ }^{\dagger}$ region). These two regimes correspond to high one-dimensional density (weak interaction) and low one-dimensional density (strong interaction), respectively. The significance of the combination $a L / r^{2} N$ can be understood by noting that it is the ratio of the $3 \mathrm{D}$ energy per particle, $\sim a \rho^{3 \mathrm{D}} \sim N a / r^{2} L$, to the $1 \mathrm{D}$ energy $\sim\left(\rho^{1 \mathrm{D}}\right)^{2}=(N / L)^{2}$. Physically, the main difference between the two regimes is

\footnotetext{
${ }^{\dagger}$ We call this the Girardeau-Tonks region only because many authors refer in the present context to Tonks [41]. In our opinion this should really be called the Girardeau region because it was he who first understood how to compute the spectrum of a 1D quantum-mechanical hard core gas and who understood that the Fermi-Dirac wave functions played a role [12]. Tonks was interested in the positive temperature partition functions of a hard core classical gas - a very different and much simpler question.
} 
that for strong interactions the motion of the particles in the longitudinal direction is highly correlated, while in the weak interaction regime it is not. Mathematically, this distinction also shows up in our proofs.

In both regimes the internal energy of the gas is small compared to the energy of confinement which is of order $N / r^{2}$. However, this in itself does not imply a specifically one-dimensional behavior. (If $a$ is sufficiently small it is satisfied in a trap of any shape.) One-dimensional behavior, when it occurs, manifests itself by the fact that the transverse motion of the atoms is uncorrelated while the longitudinal motion is correlated (very roughly speaking) in the same way as pearls on a necklace. Thus, the true criterion for 1D behavior is that $a L / r^{2} N$ is of the order unity and not merely the condition that the energy of confinement dominates the internal energy.

The starting point for our investigations is the Hamiltonian for $N$ spinless Bosons in a confining $3 \mathrm{D}$ trap potential and with a short range, repulsive pair interaction. We find it convenient to write the Hamiltonian in the following way (in appropriate units):

$$
H_{N, L, r, a}=\sum_{j=1}^{N}\left(-\Delta_{j}+V_{r}^{\perp}\left(\mathbf{x}_{j}^{\perp}\right)+V_{L}\left(z_{j}\right)\right)+\sum_{1 \leq i<j \leq N} v_{a}\left(\left|\mathbf{x}_{i}-\mathbf{x}_{j}\right|\right)
$$

with $\mathbf{x}=(x, y, z)=\left(\mathbf{x}^{\perp}, z\right)$,

$$
V_{r}^{\perp}\left(\mathbf{x}^{\perp}\right)=\frac{1}{r^{2}} V^{\perp}\left(\mathbf{x}^{\perp} / r\right), \quad V_{L}(z)=\frac{1}{L^{2}} V(z / L), \quad v_{a}(|\mathbf{x}|)=\frac{1}{a^{2}} v(|\mathbf{x}| / a),
$$

where $r, L, a$ are variable scaling parameters while $V^{\perp}, V$ and $v$ are fixed. The interaction potential $v$ is supposed to be nonnegative, of finite range and have scattering length 1 ; the scaled potential $v_{a}$ then has scattering length $a$. The external trap potentials $V$ and $V^{\perp}$ confine the motion in the longitudinal $(z)$ and the transversal $\left(\mathbf{x}^{\perp}\right)$ directions, respectively, and are assumed to be locally bounded and tend to $\infty$ as $|z|$ and $\left|\mathbf{x}^{\perp}\right|$ tend to $\infty$. To simplify the discussion we find it also convenient to assume throughout that $V$ is homogeneous of some order $s>0$, namely $V(z)=|z|^{s}$, but weaker assumptions, e.g. asymptotic homogeneity [28], would in fact suffice. The case of a simple box with hard walls is realized by taking $s=\infty$, while the usual harmonic approximation is $s=2$. Moreover, to avoid unnecessary technicalities we shall assume that $V^{\perp}$ is polynomially bounded at infinity, but our results certainly also hold for faster growing potentials, or even finite domains with Dirichlet boundary conditions. Units are chosen so that $\hbar=1$ and $2 m=1$. It is understood that the lengths associated with the ground states of $-d^{2} / d z^{2}+V(z)$ and $-\Delta^{\perp}+V^{\perp}\left(\mathbf{x}^{\perp}\right)$ are both of the order 1 so that $L$ and $r$ measure, respectively, the longitudinal and the transverse extensions of the trap. We denote the ground state energy of (1.1) by $E^{\mathrm{QM}}(N, L, r, a)$ and the ground state particle density by $\rho_{N, L, r, a}^{\mathrm{QM}}(\mathbf{x})$.

In parallel with the three-dimensional Hamiltonian we consider the Hamiltonian for $n$ Bosons in one dimension with delta interaction and coupling constant $g \geq 0$, i.e.,

$$
H_{n, g}^{1 \mathrm{D}}=\sum_{j=1}^{n}-\partial_{j}^{2}+g \sum_{1 \leq i<j \leq n} \delta\left(z_{i}-z_{j}\right)
$$


where $\partial_{j}=\partial / \partial z_{j}$. We consider this Hamiltonian for the $z_{j}$ in an interval of length $\ell$ in the thermodynamic limit, $\ell \rightarrow \infty, n \rightarrow \infty$ with $\rho=n / \ell$ fixed. The ground state energy per particle in this limit is independent of boundary conditions and can, according to [22], be written as

$$
e_{0}^{1 \mathrm{D}}(\rho)=\rho^{2} e(g / \rho),
$$

with a function $e(t)$ determined by a certain integral equation. Its asymptotic form is $e(t) \approx \frac{1}{2} t$ for $t \ll 1$ and $e(t) \rightarrow \pi^{2} / 3$ for $t \rightarrow \infty$. Thus

$$
e_{0}^{1 \mathrm{D}}(\rho) \approx \frac{1}{2} g \rho \text { for } g / \rho \ll 1
$$

and

$$
e_{0}^{1 \mathrm{D}}(\rho) \approx \frac{\pi^{2}}{3} \rho^{2} \text { for } g / \rho \gg 1 .
$$

Taking $\rho e_{0}^{1 \mathrm{D}}(\rho)$ as a local energy density for an inhomogeneous one-dimensional system we can form the energy functional

$$
\mathcal{E}[\rho]=\int_{\mathbb{R}}\left(|\nabla \sqrt{\rho}(z)|^{2}+V_{L}(z) \rho(z)+\rho(z)^{3} e(g / \rho(z))\right) d z
$$

with ground state energy defined by minimizing $\mathcal{E}[\rho]$ over all normalized densities $\rho$, i.e.,

$$
E(N, L, g)=\inf \left\{\mathcal{E}[\rho]: \rho \geq 0, \int_{\mathbb{R}} \rho(z) d z=N\right\}
$$

By standard methods (cf., e.g., [27]) one can show that there is a unique minimizer, i.e., a density $\rho_{N, L, g}(z)$ with $\int \rho_{N, L, g}(z) d z=N$ and $\mathcal{E}\left[\rho_{N, L, g}\right]=E(N, L, g)$. Here it is important to note that $t \mapsto t^{3} e(1 / t)$ is convex. We define the mean $1 D$ density of this minimizer to be

$$
\bar{\rho}=\frac{1}{N} \int_{\mathbb{R}}\left(\rho_{N, L, g}(z)\right)^{2} d z
$$

In a rigid box, i.e., for $s=\infty, \bar{\rho}$ is simply $N / L$, but in more general traps it depends also on $g$ besides $N$ and $L$. The order of magnitude of $\bar{\rho}$ in the various parameter regions will be described in the next section.

Our main result relates the $3 \mathrm{D}$ ground state energy of $(1.1), E^{\mathrm{QM}}(N, L, r, a)$, to the 1D density functional energy $E(N, L, g)$ for a suitable $g$ in the large $N$ limit provided $r / L$ and $a / r$ are sufficiently small. To state this precisely, let $e^{\perp}$ and $b\left(\mathbf{x}^{\perp}\right)$ denote the ground state energy and the normalized, nonnegative ground state wave function of $-\Delta^{\perp}+V^{\perp}\left(\mathbf{x}^{\perp}\right)$, respectively. The corresponding quantities for $-\Delta^{\perp}+V_{r}^{\perp}\left(\mathbf{x}^{\perp}\right)$ are $e^{\perp} / r^{2}$ and $b_{r}\left(\mathbf{x}^{\perp}\right)=(1 / r) b\left(\mathbf{x}^{\perp} / r\right)$. In the case that the trap is a cylinder with hard walls $b$ is a Bessel function; for a quadratic $V^{\perp}$ it is a Gaussian. In any case, $b$ is a bounded function and, in particular, $b \in L^{4}\left(\mathbb{R}^{2}\right)$. Hence we can define $g$ by

$$
g=\frac{8 \pi a}{r^{2}} \int_{\mathbb{R}^{2}}\left|b\left(\mathbf{x}^{\perp}\right)\right|^{4} d^{2} \mathbf{x}^{\perp}=8 \pi a \int_{\mathbb{R}^{2}}\left|b_{r}\left(\mathbf{x}^{\perp}\right)\right|^{4} d^{2} \mathbf{x}^{\perp} .
$$

Our main Theorem is: 
Theorem 1.1 (From 3D to 1D). Let $N \rightarrow \infty$ and simultaneously $r / L \rightarrow 0$ and $a / r \rightarrow 0$ in such a way that $r^{2} \bar{\rho} \cdot \min \{\bar{\rho}, g\} \rightarrow 0$. Then

$$
\lim \frac{E^{\mathrm{QM}}(N, L, r, a)-N e^{\perp} / r^{2}}{E(N, L, g)}=1 .
$$

Note that because of (1.5) and (1.6) the condition $r^{2} \bar{\rho} \cdot \min \{\bar{\rho}, g\} \rightarrow 0$ is the same as

$$
e_{0}^{1 \mathrm{D}}(\bar{\rho}) \ll 1 / r^{2},
$$

i.e., the average energy per particle associated with the longitudinal motion should be much smaller than the energy gap between the ground and first excited state of the confining Hamiltonian in the transverse directions. (The precise meaning of $\ll$ is that the ratio of the left side to the right side tends to zero in the limit considered.) Note also that while the one-dimensional density can be either high or low (compared to $g$ ), the gas is always dilute in a three-dimensional sense in the limit considered, i.e., $a^{3} \rho^{3 \mathrm{D}} \sim a^{2} g \bar{\rho} \ll 1$.

The two regimes mentioned previously correspond to specific restrictions on the size of the ratio $g / \bar{\rho}$ as $N \rightarrow \infty$, namely $g / \bar{\rho} \ll 1$ for the limit of the 3D GrossPitaevskii regime (weak interaction/high $1 \mathrm{D}$ density), and $g / \bar{\rho}>0$ for the 'true' one-dimensional regime (strong interaction/low 1D density). We shall now describe briefly the finer division of these regimes into five regions altogether. Three of them (Regions 1-3) belong to the weak interaction regime and two (Regions 4-5) to the strong interaction regime. In each of these regions the general functional (1.7) can be replaced by a different, simpler functional, and the energy $E(N, L, g)$ in Theorem 1.1 by the ground state energy of that functional.

The five regions are

- Region 1, the Ideal Gas case: $g / \bar{\rho} \ll N^{-2}$, with $\bar{\rho} \sim N / L$, corresponding to a non-interacting gas in an external potential.

- Region 2, the 1D GP case: $g / \bar{\rho} \sim N^{-2}$, with $\bar{\rho} \sim N / L$, described by a 1D Gross-Pitaevskii energy functional with energy density $\frac{1}{2} g \rho^{2}$.

- Region 3, the 1D TF case: $N^{-2} \ll g / \bar{\rho} \ll 1$, with $\bar{\rho} \sim(N / L)(N g L)^{-1 /(s+1)}$, where $s$ is the degree of homogeneity of the longitudinal confining potential $V$. This region is described by a Thomas-Fermi type functional with energy density $\frac{1}{2} g \rho^{2}$, without a gradient term.

- Region 4, the LL case: $g / \bar{\rho} \sim 1$, with $\bar{\rho} \sim(N / L) N^{-2 /(s+2)}$, described by an energy functional with the Lieb-Liniger energy (1.4), without a gradient term.

- Region 5, the GT case: $g / \bar{\rho} \gg 1$, with $\bar{\rho} \sim(N / L) N^{-2 /(s+2)}$, described by a functional with energy density $\sim \rho^{3}$, corresponding to the Girardeau-Tonks limit of the LL energy density.

We note that the condition $g / \bar{\rho} \sim 1$ means that Region 4 requires the gas cloud to have aspect ratio $r / \bar{L}$ of the order $N^{-1}(a / r)$ or smaller, where $\bar{L} \equiv N / \bar{\rho} \sim L N^{2 /(s+2)}$ 
is the length of the cloud. Experimentally, such small aspect ratios are quite a challenge and the situations described in $[4,15,16,38]$ are still rather far from this regime. It may not be completely out of reach, however.

The condition $a / r \rightarrow 0$ is automatically fulfilled in Regions $1-4$, provided (1.12) holds, since $(a / r)^{2}=r^{2}(g \bar{\rho})(g / \bar{\rho})=r^{2} \bar{\rho}^{2}(g / \bar{\rho})^{2} \ll 1$ by (1.12) if $g / \bar{\rho}$ is bounded. Moreover, as discusssed in the next section, in Regions 1-2 the condition $r / L \rightarrow 0$ implies (1.12) and hence $a / r \rightarrow 0$, and in Region $4, a / r \rightarrow 0$ implies (1.12). The hypotheses of Theorem 1.1 are thus not entirely independent.

In the next Section 2 we define the various energy functionals more precisely and also discuss the $1 \mathrm{D}$ behavior of the density $\rho_{N, L, r, a}^{\mathrm{QM}}(\mathbf{x})$, separately for each region. Moreover, we prove, in Subsection 2.7, that Regions 1-3 can be reached as limiting cases of a 3D Gross-Pitaevskii theory. In this sense, the behavior in these regions contains remnants of the 3D theory, which also shows up in the fact that BEC prevails in Regions 1 and 2, as discussed in Section 5. Heuristically, these traces of 3D can be understood from the fact that in Regions 1-3 the 1D formula for the energy per particle $g \bar{\rho} \sim a N /\left(r^{2} \bar{L}\right)$, gives the same result as the three-dimensional formula [31], i.e., scattering length times three-dimensional density. This is no longer so in Regions 4 and 5 and different mathematical methods are required.

Despite significant differences the proof of Theorem 1.1 has some basic strategies in common with the (considerably simpler) proof of the Gross-Pitaevskii limit Theorem in [27] (see also [31] and [26]). The upper bound for the energy in Regions 1-3, given in Subsection 4.1, is the simplest estimate and can be obtained by a method analogous to that of [27]. For the lower bound, and also for the upper bound in Regions $4-5$, one considers first finite numbers of particles in boxes with Neumann or Dirichlet boundary conditions, and subsequently puts these boxes together to treat inhomogeneous external potentials and the infinite particle number limit. For the lower bound of the energy in the boxes Dyson's Lemma [9, 31, 26], which converts a "hard" potential into a "soft" potential at the expense of sacrificing kinetic energy, is an essential tool. The main differences compared to [27] are on the one hand due to the fact that in Regions 1-3 the lower bounds in [27] are not valid because they are not uniform in the shape of the trap, and on the other hand due to the correlations in the Lieb-Liniger wave function for the longitudinal motion in Regions 4-5. For the latter reason even the proof of the upper bound in Regions $4-5$ is considerably more involved than for Regions 1-3.

Section 3 contains the main technical estimates for the boxes. We consider here the 3D Hamiltonian for a finite number of particles on a finite interval in the longitudinal direction with Neumann or Dirichlet boundary conditions and a confining potential $V_{r}^{\perp}$ in the transverse directions. We estimate its energy from above and below in terms of the energy of a 1D Hamiltonian with delta interactions. In Section 4 we apply these results to prove Theorem 1.1. In the last Section 5 we consider the question of Bose-Einstein condensation and prove that it holds in Regions 1 and 2.

Part of the results of this work were announced in [29]. 


\section{The five parameter regions}

We shall now discuss the simplifications of Theorem 1.1 in the five different parameter regions. Besides the ground state energy we also consider the convergence of the quantum mechanical density $\rho_{N, L, r, a}^{\mathrm{QM}}(\mathbf{x})$ averaged over the transverse variables, i.e., of

$$
\hat{\rho}_{N, L, r, a}^{\mathrm{QM}}(z):=\int \rho_{N, L, r, a}^{\mathrm{QM}}\left(\mathbf{x}^{\perp}, z\right) d^{2} \mathbf{x}^{\perp} .
$$

We state the results in the form of five theorems, one for each region. These theorems will be proved in Section 4. Together they imply Theorem 1.1 because the energy functionals involved are all limiting cases of the general functional (1.8). The methods for proving the latter statement are fairly standard (cf., e.g., [27, 28] for similar computations). Since a proof of all five limit theorems for the functional (1.8) would be largely repetitious, we shall limit ourselves to giving a proof of two of them in Subsection 2.6, as an example.

\subsection{The Ideal Gas Region}

This region corresponds to the trivial case where the interaction is so weak that it effectively vanishes in the large $N$ limit and everything collapses to the ground state of $-d^{2} / d z^{2}+V_{L}(z)$. By scaling the ground state energy and wave function of this latter operator can be written as $L^{-2} e^{\|}$and $L^{-1 / 2} \sqrt{\rho^{\|}(z / L)}$ where $e^{\|}$and $\sqrt{\rho^{\|}(z)}$ are the corresponding quantities for $-d^{2} / d z^{2}+V(z)$.

Theorem 2.1 (Ideal gas limit). Suppose $r / L \rightarrow 0$ and $N g L \sim N a L / r^{2} \rightarrow 0$ as $N \rightarrow \infty$. Then

$$
\left(N / L^{2}\right)^{-1}\left(E^{\mathrm{QM}}(N, L, a, r)-N e^{\perp} / r^{2}\right) \rightarrow e^{\|}
$$

and

$$
(N / L)^{-1} \hat{\rho}_{N, L, r, a}^{\mathrm{QM}}(L z) \rightarrow \rho^{\|}(z)
$$

weakly in $L^{1}(\mathbb{R})$.

\subsection{The 1D Gross-Pitaevskii Region}

This region is described by the 1D GP density functional

$$
\mathcal{E}_{L, g}^{\mathrm{GP}}[\rho]=\int_{\mathbb{R}}\left(|\nabla \sqrt{\rho}(z)|^{2}+V_{L}(z) \rho(z)+\frac{1}{2} g \rho(z)^{2}\right) d z
$$

corresponding to the high density approximation (1.5) of the interaction energy in (1.7). Its ground state energy

$$
E^{\mathrm{GP}}(N, L, g)=\inf \left\{\mathcal{E}_{L, g}^{\mathrm{GP}}[\rho]: \rho \geq 0, \int_{\mathbb{R}} \rho(z) d z=N\right\}
$$

has the scaling property

$$
E^{\mathrm{GP}}(N, L, g)=\left(N / L^{2}\right) E^{\mathrm{GP}}(1,1, N g L)
$$


and likewise, the minimizer $\rho_{N, L, g}^{\mathrm{GP}}(z)$ satisfies

$$
\rho_{N, L, g}^{\mathrm{GP}}(L z)=(N / L) \rho_{1,1, N g L}^{\mathrm{GP}}(z) .
$$

Theorem 2.2 (1D GP limit). Suppose $r / L \rightarrow 0$ and $N g L \sim N a L / r^{2}$ is fixed as $N \rightarrow \infty$. Then

$$
\left(N / L^{2}\right)^{-1}\left(E^{\mathrm{QM}}(N, L, a, r)-N e^{\perp} / r^{2}\right) \rightarrow E^{\mathrm{GP}}(1,1, N g L)
$$

and

$$
(N / L)^{-1} \hat{\rho}_{N, L, r, a}^{\mathrm{QM}}(L z) \rightarrow \rho_{1,1, N g L}^{\mathrm{GP}}(z)
$$

weakly in $L^{1}(\mathbb{R})$.

Remark. If $r / L \rightarrow 0$ and $N g L$ stays bounded, as in Regions 1 and 2, condition (1.12) is automatically satisfied, because $g \bar{\rho} r^{2} \sim a N / L \sim(r / L)^{2}(N g L)$. Likewise, $a / r \rightarrow 0$, because $a / r=(r / L) N^{-1}(N g L)$.

\subsection{The 1D 'Thomas-Fermi' Region}

This region is a limiting case of the previous one in the sense that $N g L \sim N a L / r^{2} \rightarrow$ $\infty$, but $a / r$ is sufficiently small so that $g / \bar{\rho} \sim\left(a L / N r^{2}\right)\left(N a L / r^{2}\right)^{1 /(s+1)} \rightarrow 0$, i.e., the high density approximation in (1.5) is still valid. Here $s$ is the degree of homogeneity of $V$ and the explanation of the factor $\left(N a L / r^{2}\right)^{1 /(s+1)} \sim(N g L)^{1 /(s+1)}$ is as follows: The linear extension $\bar{L}$ of the minimizing density $\rho_{N, L, g}^{\mathrm{GP}}$ is for large values of $N g L$ determined by $V_{L}(\bar{L}) \sim g(N / \bar{L})$, which gives $\bar{L} \sim(N g L)^{1 /(s+1)} L$. In addition condition (1.12) requires $g \bar{\rho} \ll r^{-2}$, which means that $(N a / L)\left(N a L / r^{2}\right)^{-1 /(s+1)} \rightarrow 0$.

If $N g L \sim N a L / r^{2} \rightarrow \infty$ the gradient term in the functional (2.5) becomes negligible compared to the other terms. In fact, by a simple scaling,

$\mathcal{E}_{L, g}^{\mathrm{GP}}[\rho]=\frac{N}{L^{2}}(N g L)^{s /(s+1)} \int_{\mathbb{R}}\left((N g L)^{-(s+2) /(s+1)}|\nabla \sqrt{\tilde{\rho}(z)}|^{2}+V(z) \tilde{\rho}(z)+\frac{1}{2} \tilde{\rho}(z)^{2}\right) d z$

where the scaled density $\tilde{\rho}$ is determined by

$$
\rho(z)=\left(N / \bar{L}_{\mathrm{TF}}\right) \tilde{\rho}\left(z / \bar{L}_{\mathrm{TF}}\right), \quad \text { with } \bar{L}_{\mathrm{TF}}:=(N g L)^{1 /(s+1)} L .
$$

This leads to the functional

$$
\mathcal{E}_{L, g}^{\mathrm{TF}}[\rho]=\int_{\mathbb{R}}\left(V_{L}(z) \rho(z)+\frac{1}{2} g \rho(z)^{2}\right) d z
$$

whose ground state energy

$$
E^{\mathrm{TF}}(N, L, g)=\inf \left\{\mathcal{E}_{L, g}^{\mathrm{TF}}[\rho]: \rho \geq 0, \int_{\mathbb{R}} \rho(z) d z=N\right\}
$$

has the scaling property

$$
E^{\mathrm{TF}}(N, L, g)=\left(N / L^{2}\right)(N g L)^{s /(s+1)} E^{\mathrm{TF}}(1,1,1) .
$$


The minimizer $\rho_{N, L, g}^{\mathrm{TF}}$ satisfies

$$
\rho_{N, L, g}^{\mathrm{TF}}\left(\bar{L}_{\mathrm{TF}} z\right)=\left(N / \bar{L}_{\mathrm{TF}}\right) \rho_{1,1,1}^{\mathrm{TF}}(z)
$$

and can be computed explicitly:

$$
\rho_{1,1,1}^{\mathrm{TF}}(z)=\left[\mu^{\mathrm{TF}}-V(z)\right]_{+}
$$

where $[t]_{+}=\max \{t, 0\}$ and $\mu^{\mathrm{TF}}$ is determined by the normalization $\int \rho_{1,1,1}^{\mathrm{TF}}(z) d z=1$.

Because of a formal similarity with the Thomas-Fermi energy functional for fermions, which also has no gradient terms, the functional (2.12) is in the literature usually referred to as a 'Thomas-Fermi' functional, but the physics is, of course, quite different.

The limit theorem in this region is

Theorem 2.3 (1D 'TF limit'). Suppose, as $N \rightarrow \infty, r / L \rightarrow 0, N g L \sim N a L / r^{2} \rightarrow$ $\infty$, but $g / \bar{\rho} \sim\left(a \bar{L}_{\mathrm{TF}} / N r^{2}\right) \rightarrow 0$ and $g \bar{\rho} r^{2} \sim N a / \bar{L}_{\mathrm{TF}} \rightarrow 0$ with $\bar{L}_{\mathrm{TF}}$ given in (2.11). Then

$$
\left(N / L^{2}\right)^{-1}(N g L)^{-s /(s+1)}\left[E^{\mathrm{QM}}(N, L, a, r)-N e^{\perp} / r^{2}\right] \rightarrow E^{\mathrm{TF}}(1,1,1)
$$

and

$$
\left(N / \bar{L}_{\mathrm{TF}}\right)^{-1} \hat{\rho}_{N, L, r, a}^{\mathrm{QM}}\left(\bar{L}_{\mathrm{TF}} z\right) \rightarrow \rho_{1,1,1}^{\mathrm{TF}}(z)
$$

weakly in $L^{1}(\mathbb{R})$.

\subsection{The Lieb-Liniger Region}

This region corresponds to the case $g / \bar{\rho} \sim 1$, so that neither the high density (1.5) nor the low density approximation (1.6) is valid and the full LL energy (1.4) has to be used. The extension $\bar{L}$ of the system is now determined by $V_{L}(\bar{L}) \sim(N / \bar{L})^{2}$ which leads to $\bar{L}_{\mathrm{LL}}=L N^{2 /(s+2)}$, in contrast to $\bar{L}_{\mathrm{TF}}=L(N g L)^{1 /(s+1)}$ in the TF case. Condition (1.12) means in this region that $N r / \bar{L}_{\mathrm{LL}} \sim N^{s /(s+2)} r / L \rightarrow 0$. Since $N r / \bar{L}_{\mathrm{LL}} \sim(\bar{\rho} / g)(a / r)$, this condition is automatically fulfilled if $g / \bar{\rho}$ is bounded away from zero and $a / r \rightarrow 0$. Conversely, if $g / \bar{\rho}$ is bounded, then (1.12) implies $a / r \rightarrow 0$.

The energy functional is

$$
\mathcal{E}_{L, g}^{\mathrm{LL}}[\rho]=\int_{\mathbb{R}}\left(V_{L}(z) \rho(z)+\rho(z)^{3} e(g / \rho(z))\right) d z
$$

with corresponding energy

$$
E^{\mathrm{LL}}(N, L, g)=\inf \left\{\mathcal{E}_{L, g}^{\mathrm{LL}}[\rho]: \rho \geq 0, \int_{\mathbb{R}} \rho(z) d z=N\right\}
$$

Introducing the density parameter

$$
\gamma:=N / \bar{L}_{\mathrm{LL}}=(N / L) N^{-2 /(s+2)}
$$


we can write the scaling relation of the functional as

$$
E^{\mathrm{LL}}(N, L, g)=N \gamma^{2} E^{\mathrm{LL}}(1,1, g / \gamma) .
$$

The minimizer, $\rho^{\mathrm{LL}}(z)$, satisfies

$$
\rho_{N, L, g}^{\mathrm{LL}}\left(\bar{L}_{\mathrm{LL}} z\right)=\gamma \rho_{1,1, g / \gamma}^{\mathrm{LL}}(z) .
$$

Theorem 2.4 (LL limit). Suppose $r / L \rightarrow 0$ and $a / r \rightarrow 0$, with $g / \gamma>0$ fixed as $N \rightarrow \infty$. Then

$$
\left(N \gamma^{2}\right)^{-1}\left[E^{\mathrm{QM}}(N, L, a, r)-N e^{\perp} / r^{2}\right] \rightarrow E^{\mathrm{LL}}(1,1, g / \gamma)
$$

and

$$
\gamma^{-1} \hat{\rho}_{N, L, r, a}^{\mathrm{QM}}\left(\bar{L}_{\mathrm{LL}} z\right) \rightarrow \rho_{1,1, g / \gamma}^{\mathrm{LL}}(z)
$$

weakly in $L^{1}(\mathbb{R})$.

\subsection{The Girardeau-Tonks Region}

This region corresponds to impenetrable particles, i.e, the limiting case $g / \bar{\rho} \rightarrow \infty$ and hence the formula (1.6) for the energy density. As in Region 4, the mean density is here $\bar{\rho} \sim \gamma=(N / L) N^{-2 /(s+2)}$. The energy functional is

$$
\mathcal{E}_{L}^{\mathrm{GT}}[\rho]=\int_{\mathbb{R}}\left(V_{L}(z) \rho(z)+\left(\pi^{2} / 3\right) \rho(z)^{3}\right) d z
$$

with energy

$$
E^{\mathrm{GT}}(N, L)=\inf \left\{\mathcal{E}_{L}^{\mathrm{GT}}[\rho]: \rho \geq 0, \int_{\mathbb{R}} \rho(z) d z=N\right\}
$$

which can be written

$$
E^{\mathrm{GT}}(N, L)=N \gamma^{2} E^{\mathrm{GT}}(1,1) .
$$

The minimizer $\rho_{1,1}^{\mathrm{GT}}(z)$ has the form

$$
\rho_{1,1}^{\mathrm{GT}}(z)=\pi^{-1}\left[\mu^{\mathrm{GT}}-V(z)\right]_{+}^{1 / 2},
$$

with $\mu^{\mathrm{GT}}$ determined by the normalization. Note that its shape is different from that of (2.16), which makes it possible to distinguish experimentally the TF regime from the GT regime. The scaling relation for the minimizer is

$$
\rho_{N, L}^{\mathrm{GT}}\left(\bar{L}_{\mathrm{LL}} z\right)=\gamma \rho_{1,1}^{\mathrm{GT}}(z) .
$$

Theorem 2.5 (GT limit). Suppose $r / L \rightarrow 0$ and $a / r \rightarrow 0$, with $g / \gamma \rightarrow \infty$ as $N \rightarrow \infty$. Then

$$
\left(N \gamma^{2}\right)^{-1}\left[E^{\mathrm{QM}}(N, L, a, r)-N e^{\perp} / r^{2}\right] \rightarrow E^{\mathrm{GT}}(1,1)
$$

and

$$
\gamma^{-1} \hat{\rho}_{N, L, r, a}^{\mathrm{QM}}\left(\bar{L}_{\mathrm{LL}} z\right) \rightarrow \rho_{1,1}^{\mathrm{GT}}(z)
$$

weakly in $L^{1}(\mathbb{R})$. 


\subsection{Limiting cases of the general energy functional}

As already stated, the proof of Theorem 1.1 in Section 4 consists in comparing the ground state energy of the many-body Hamiltonian (1.1) with the ground state energies of the functionals defined in Subsections 2.1-2.5 in the various parameter domains. To link these special cases to the functional (1.7) it then remains to show that the ground state energy of (1.7) coincides with that of the functionals in Subsections $2.1-2.5$ in the appropriate asymptotic limits. The proof of this follows the same pattern as the derivation of the 3D TF limit from 3D GP theory in $[27,28]$ and we shall here only give explicit proofs for the functionals in Theorems 2.3 and 2.4 as examples. The limit theorems for the density are derived from the energy convergence by variation of the external potential in a standard way (c.f., e.g. [28]).

Proposition 2.1. If $N \rightarrow \infty, N g L \rightarrow \infty$, but $g \bar{L}_{\mathrm{TF}} / N=g L(N g L)^{1 /(s+1)} / N \rightarrow 0$, then

$$
\left[\left(N / L^{2}\right)(N g L)^{s /(s+1)}\right]^{-1} E(N, L, g) \rightarrow E^{\mathrm{TF}}(1,1,1)
$$

and

$$
\left(N / \bar{L}_{\mathrm{TF}}\right)^{-1} \rho_{N, L, g}\left(\bar{L}_{\mathrm{TF}} z\right) \rightarrow \rho_{1,1,1}^{\mathrm{TF}}(z)
$$

weakly in $L^{1}(\mathbb{R})$.

Proof. With $\tilde{\rho}$ the scaled density given by (2.11) the energy functional (1.7) can be written

$$
\begin{aligned}
\mathcal{E}[\rho]= & \left(N / L^{2}\right)(N g L)^{s /(s+1)} \int_{\mathbb{R}}\left((N g L)^{-(s+2) /(s+1)}|\partial \sqrt{\tilde{\rho}(z)}|^{2}\right. \\
& \left.+V(z) \tilde{\rho}(z)+\tilde{\rho}(z)^{3} N\left(g \bar{L}_{\mathrm{TF}}\right)^{-1} e\left(g \bar{L}_{\mathrm{TF}} / N \tilde{\rho}(z)\right)\right) d z
\end{aligned}
$$

Now $t e(1 / t) \leq \frac{1}{2}$ for all $t[22]$, so

$$
\begin{aligned}
& {\left[\left(N / L^{2}\right)(N g L)^{s /(s+1)}\right]^{-1} \mathcal{E}[\rho]} \\
& \leq \int_{\mathbb{R}}\left((N g L)^{-(s+2) /(s+1)}|\partial \sqrt{\tilde{\rho}(z)}|^{2}+V(z) \tilde{\rho}(z)+\frac{1}{2} \tilde{\rho}(z)^{2}\right) d z .
\end{aligned}
$$

Let

$$
j_{\varepsilon}(z)=(2 \varepsilon)^{-1} \exp (-|z| / \varepsilon) .
$$

Define $\tilde{\rho}=\rho_{1,1,1}^{\mathrm{TF}} * j_{\varepsilon}$. Then, since $\left|\partial j_{\varepsilon}\right|=\varepsilon^{-1} j_{\varepsilon}$ and $\int \tilde{\rho}(z) d z=1$,

$$
\int|\partial \sqrt{\tilde{\rho}(z)}|^{2} d z \leq 1 /\left(4 \varepsilon^{2}\right)<\infty
$$

and (2.36) implies

$$
\limsup _{N \rightarrow \infty}\left[\left(N / L^{2}\right)(N g L)^{s /(s+1)}\right]^{-1} E(N, L, g) \leq \mathcal{E}_{1,1}^{\mathrm{TF}}\left[\rho_{1,1,1}^{\mathrm{TF}} * j_{\varepsilon}\right]
$$


in the limit considered. Moreover, $\int\left(\rho_{1,1,1}^{\mathrm{TF}} * j_{\varepsilon}\right)^{2} \leq \int\left(\rho_{1,1,1}^{\mathrm{TF}}\right)^{2}$ because $\int j_{\varepsilon}=1$, and

$$
\int \rho_{1,1,1}^{\mathrm{TF}} * j_{\varepsilon}(z)|z|^{s} d z \rightarrow \int \rho_{1,1,1}^{\mathrm{TF}}(z)|z|^{s} d z
$$

for $\varepsilon \rightarrow 0$. (Note that $\rho_{1,1,1}^{\mathrm{TF}}=\left[\mu^{\mathrm{TF}}-|z|^{s}\right]_{+}$is continuous and of compact support.) Hence

$$
\limsup _{N \rightarrow \infty}\left[\left(N / L^{2}\right)(N g L)^{s /(s+1)}\right]^{-1} E(N, L, g) \leq E^{\mathrm{TF}}(1,1,1) .
$$

On the other hand, dropping the positive gradient term in (2.35) gives

$$
\left[\left(N / L^{2}\right)(N g L)^{s /(s+1)}\right]^{-1} \mathcal{E}[\rho] \geq \int_{\mathbb{R}}\left(V(z) \tilde{\rho}(z)+\tilde{\rho}(z)^{3} M e(1 / M \tilde{\rho}(z))\right) d z,
$$

with $M=N /\left(g \bar{L}_{\mathrm{TF}}\right)$. Note that $M \rightarrow \infty$ in the limit considered here. The functional on the right side of (2.42) has as minimizer

$$
\rho^{(M)}(z)=\left[f_{M}^{-1}\left(\mu^{(M)}-V(z)\right)\right]_{+}
$$

where $f_{M}^{-1}$ is the inverse of the function $f_{M}(t)=d / d t\left[M t^{3} e(1 / t M)\right]$ and $\mu^{(M)}$ is chosen so that $\int \rho^{(M)}=1$. Note also that $t^{-1} f_{M}(t) \rightarrow 1$ as $M \rightarrow \infty$, uniformly on $\left[\delta, \infty\left[\right.\right.$ for every $\delta>0$. From this it follows easily that $\rho^{(M)}$ converges uniformly to $\rho_{1,1,1}^{\mathrm{TF}}$, given by $(2.16)$, as $M \rightarrow \infty$. With $\rho=\rho_{N, L, g}$, the minimizer of $\mathcal{E}$, we thus obtain from $(2.42)$

$$
\liminf _{N \rightarrow \infty}\left[\left(N / L^{2}\right)(N g L)^{s /(s+1)}\right]^{-1} E(N, L, g) \geq E^{\mathrm{TF}}(1,1,1) .
$$

To prove the corresponding result (2.34) for the density we pick a $C^{\infty}$ function $Y$ of compact support together with an $\varepsilon>0$ and replace $V_{L}(z)$ by

$$
V_{L}(z)+\frac{\varepsilon}{L^{2}}(N g L)^{s /(s+1)} Y\left(L^{-1}(N g L)^{-1 /(s+1)} z\right)=\frac{1}{L^{2}}(N g L)^{s /(s+1)}\left[V\left(z^{\prime}\right)+\varepsilon Y\left(z^{\prime}\right)\right]
$$

with $z^{\prime}=z / \bar{L}_{\mathrm{TF}}=L^{-1}(N g L)^{-1 /(s+1)} z$. While $V\left(z^{\prime}\right)+\varepsilon Y\left(z^{\prime}\right)$ is not strictly homogeneous of order $s$, it is asymptotically homogeneous in the sense of Def. 1.1 in [28] and as in the proof of Lemma 2.3 in [28] this is sufficient for (2.33), now with the modified external potential (2.45). Since both (1.8) and the TF energy are concave in $\varepsilon$, the derivative with respect to $\varepsilon$ can be exchanged with the limits $N \rightarrow \infty$, $(N g L) \rightarrow \infty$, giving (2.34) in the sense of distributions. Since the densities have norm 1 , the convergence holds also weakly in $L^{1}(\mathbb{R})$.

Proposition 2.2. If $N \rightarrow \infty$ with $g / \gamma$ fixed, where $\gamma=N / \bar{L}_{\mathrm{LL}}=(N / L) N^{-2 /(s+2)}$, then

$$
\left(N \gamma^{2}\right)^{-1} E(N, L, g) \rightarrow E^{\mathrm{LL}}(1,1, g / \gamma)
$$

and

$$
\gamma^{-1} \rho_{N, L, g}\left(\bar{L}_{\mathrm{LL}} z\right) \rightarrow \rho_{1,1, g / \gamma}^{\mathrm{LL}}(z)
$$

weakly in $L^{1}(\mathbb{R})$. 
Proof. With $\bar{L}_{\mathrm{LL}}=L N^{2 /(s+2)}$ we define the scaled density $\tilde{\rho}$ by

$$
\rho(z)=\left(N / \bar{L}_{\mathrm{LL}}\right) \tilde{\rho}\left(z / \bar{L}_{\mathrm{LL}}\right) .
$$

The energy functional (1.7) can then be written

$$
\mathcal{E}[\rho]=N \gamma^{2} \int_{\mathbb{R}}\left[N^{-2}|\partial \sqrt{\tilde{\rho}(z)}|^{2}+V(z) \tilde{\rho}(z)+\tilde{\rho}(z)^{3} e(g /(\gamma \tilde{\rho}(z)))\right] d z .
$$

The lower bound

$$
\left(N \gamma^{2}\right)^{-1} E(N, L, g) \geq E^{\mathrm{LL}}(1,1, g / \gamma)
$$

follows simply by dropping the positive gradient term from the right side of (2.49).

For the upper bound take $j_{\varepsilon}$ as in (2.37) and define $\tilde{\rho}=j_{\varepsilon} * \rho_{1,1, g / \gamma}^{\mathrm{LL}}$ to obtain

$$
\left(N \gamma^{2}\right)^{-1} \mathcal{E}[\rho] \leq \frac{N^{-2}}{4 \varepsilon^{2}}+\mathcal{E}_{1,1, g / \gamma}^{\mathrm{LL}}[\tilde{\rho}]
$$

and hence

$$
\limsup _{N \rightarrow \infty}\left(N \gamma^{2}\right)^{-1} E(N, L, g) \leq \mathcal{E}_{1,1, g / \gamma}^{\mathrm{LL}}\left[j_{\varepsilon} * \rho_{1,1, g / \gamma}^{\mathrm{LL}}\right]
$$

for all $\varepsilon>0$. The convergence

$$
\lim _{\varepsilon \rightarrow 0} \mathcal{E}_{1,1, g / \gamma}^{\mathrm{LL}}\left[j_{\varepsilon} * \rho_{1,1, g / \gamma}^{\mathrm{LL}}\right]=\mathcal{E}_{1,1, g / \gamma}^{\mathrm{LL}}\left[\rho_{1,1, g / \gamma}^{\mathrm{LL}}\right]=E^{\mathrm{LL}}(1,1, g / \gamma)
$$

follows by continuity of $|z|^{s}$ and $t^{2} e(t)$ and uniform convergence of $j_{\varepsilon} * \rho_{1,1, g / \gamma}^{\mathrm{LL}}$ to $\rho_{1,1, g / \gamma}^{\mathrm{LL}}$.

The convergence of the densities follows as in the previous proposition by perturbing the external potential, this time replacing $V_{L}(z)$ by

$$
V_{L}(z)+\varepsilon \gamma^{2} Y\left(L^{-1} N^{-2 /(s+2)} z\right)=\gamma^{2}\left[V\left(z^{\prime}\right)+\varepsilon Y\left(z^{\prime}\right)\right]
$$

with $z^{\prime}=z / \bar{L}_{\mathrm{LL}}=L^{-1} N^{-2 /(s+2)} z$.

\subsection{One-dimensional GP as limit of three-dimensional GP}

We shall now demonstrate that the ground state energy in Regions 1-3 can be obtained as a limit of the three-dimensional Gross-Pitaevskii energy. The latter is defined by the energy functional

$$
\mathcal{E}_{3 \mathrm{D}}^{\mathrm{GP}}[\Phi]=\int_{\mathbb{R}^{3}}\left(|\nabla \Phi(\mathbf{x})|^{2}+\left\{V_{r}^{\perp}\left(\mathbf{x}^{\perp}\right)+V_{L}(z)\right\}|\Phi(\mathbf{x})|^{2}+4 \pi a|\Phi(\mathbf{x})|^{4}\right) d^{3} \mathbf{x} .
$$

We denote its ground state energy, i.e, the infimum over all $\Phi$ with $\int|\Phi|^{2}=N$, by $E_{3 \mathrm{D}}^{\mathrm{GP}}(N, L, r, a)$. It satisfies the scaling relation

$$
E_{3 \mathrm{D}}^{\mathrm{GP}}(N, L, r, a)=\left(N / L^{2}\right) E_{3 \mathrm{D}}^{\mathrm{GP}}(1,1, r / L, N a / L) .
$$

Because of (2.56) and (2.6) it is sufficient to compare $E_{3 \mathrm{DP}}^{\mathrm{GP}}$ and $E^{\mathrm{GP}}$ for $N=1$ and $L=1$. 
Theorem 2.6. Let $g$ be given by (1.10). In the limit $r \rightarrow 0$ and $a \rightarrow 0$,

$$
\frac{E_{3 \mathrm{D}}^{\mathrm{GP}}(1,1, r, a)-e^{\perp} / r^{2}}{E^{\mathrm{GP}}(1,1, g)} \rightarrow 1,
$$

uniformly in $g$ as long as $r^{2} E^{\mathrm{GP}}(1,1, g) \rightarrow 0$.

Proof. We denote the minimizer of the one-dimensional GP functional (2.4) with $N=1, L=1$ and $g$ fixed by $\phi(z)^{2}$. Taking $b_{r}\left(\mathbf{x}^{\perp}\right) \phi(z)$ as trial function for the 3D functional (2.55) and using the definition (1.10) of $g$ we obtain without further ado the upper bound

$$
E_{3 \mathrm{D}}^{\mathrm{GP}}(1,1, r, a) \leq e^{\perp} / r^{2}+E^{\mathrm{GP}}(1,1, g)
$$

for all $r$ and $a$. For a lower bound we consider the one-particle Hamiltonian

$$
H_{r, a}=-\Delta^{\perp}+V_{r}^{\perp}\left(\mathbf{x}^{\perp}\right)-\partial_{z}^{2}+V(z)+8 \pi a b_{r}\left(\mathbf{x}^{\perp}\right)^{2} \phi(z)^{2} .
$$

Taking the 3D Gross-Pitaevskii minimizer $\Phi(\mathbf{x})$ for $N=1, L=1$, as trial function we get

$$
\begin{aligned}
\inf \operatorname{spec} H_{r, a} & \leq E_{3 \mathrm{D}}^{\mathrm{GP}}(1,1, r, a)-4 \pi a \int \Phi^{4}+8 \pi a \int b_{r}^{2} \phi^{2} \Phi^{2} \\
& \leq E_{3 \mathrm{D}}^{\mathrm{GP}}(1,1, r, a)+4 \pi a \int b_{r}^{4} \phi^{4} \\
& =E_{3 \mathrm{D}}^{\mathrm{GP}}(1,1, r, a)+\frac{g}{2} \int \phi^{4} .
\end{aligned}
$$

On the other hand, inf spec $H_{r, a}$ can be bounded below by Temple's inequality [40], which says that for any Hamiltonian $H$ with lowest eigenvalues $E_{0}<E_{1}$ and expectation value $\langle H\rangle<E_{1}$ in some state,

$$
E_{0} \geq\langle H\rangle-\frac{\left\langle(H-\langle H\rangle)^{2}\right\rangle}{E_{1}-\langle H\rangle} .
$$

We apply this to $H=H_{r, a}$ and the state defined by $b_{r} \phi$. Here

$$
\langle H\rangle=\frac{e^{\perp}}{r^{2}}+E^{\mathrm{GP}}(1,1, g)+\frac{g}{2} \int \phi^{4},
$$

and since $E_{1} \geq \tilde{e}^{\perp} / r^{2}$ with $\tilde{e}^{\perp}>e^{\perp},(2.62)$ is smaller than $E_{1}$ for $r^{2} E^{\mathrm{GP}}(1,1, g)$ small enough. Moreover,

$$
(H-\langle H\rangle)\left(b_{r} \phi\right)=\left(8 \pi a b_{r}^{2}-g\right) \phi^{3} b_{r}
$$

and thus

$$
\begin{aligned}
\left\langle(H-\langle H\rangle)^{2}\right\rangle & =\left(\int \phi^{6}\right)\left[(8 \pi a)^{2}\left(\int b_{r}^{6}\right)-g^{2}\right] \leq\left(\int \phi^{6}\right)(8 \pi a)^{2}\left(\int b_{r}^{4}\right)\left\|b_{r}\right\|_{\infty}^{2} \\
& \leq \text { const. } g\|\phi\|_{\infty}^{2} g \int \phi^{4} \leq \text { const. } E^{\mathrm{GP}}(1,1, g)^{2}
\end{aligned}
$$


where we used [28, Lemma 2.1] to bound $g\|\phi\|_{\infty}^{2}$ by the GP energy. Combining (2.60), (2.61) and (2.62) we thus get

$$
E_{3 \mathrm{DP}}^{\mathrm{GP}}(1,1, r, a)-e^{\perp} / r^{2} \geq E^{\mathrm{GP}}(1,1, g)\left(1-\text { const. } r^{2} E^{\mathrm{GP}}(1,1, g)\right)
$$

and the proof is complete.

Remark. In combination with Theorem 2.2 this results demonstrates a fortiori that the three-dimensional GP limit theorem in [27] holds uniformly in $r / L$, provided $N a L / r^{2}$ stays bounded. A more direct proof of this fact, closer to the lines of [27], is certainly possible, but it requires redoing all estimates keeping track of the dependence on $r / L$.

\section{$3 \quad$ Finite $n$ bounds}

Before we give the proof of our main Theorem 1.1 in Section 4, we will explain briefly the strategy, and give some auxiliary results in this section. In particular, we will derive upper and lower bounds on the ground state energy of (1.1) with the external potential $V_{L}(z)$ replaced by a box with Dirichlet and Neumann boundary conditions, respectively. To obtain bounds on the full Hamiltonian (1.1), space will be divided in $z$-direction into small boxes of side length $\ell$, and the bounds of this section will be used in every box. The reason for this is twofold: first, this allows to consider an essentially homogeneous systems, without the additional difficulty of the external potential $V_{L}(z)$, and secondly, by varying $\ell$ the particle number in each box can be controlled. This is necessary, since the bounds we obtain in every box will not be uniform in the particle number.

Since the particle number in the boxes will be small (compared to $N$ ), we denote it by $n$. In this section, we study the Hamiltonian

$$
H=\sum_{j=1}^{n}\left(-\Delta_{j}+V_{r}^{\perp}\left(\mathbf{x}_{j}^{\perp}\right)\right)+\sum_{1 \leq i<j \leq n} v_{a}\left(\left|\mathbf{x}_{i}-\mathbf{x}_{j}\right|\right)
$$

on $L^{2}\left(\left(\mathbb{R}^{2} \times[0, \ell]\right)^{n}\right)$. Let $E_{\mathrm{D}}^{\mathrm{QM}}(n, \ell, r, a)$ and $E_{\mathrm{N}}^{\mathrm{QM}}(n, \ell, r, a)$ denote its ground state energy with Dirichlet and Neumann boundary conditions, respectively. The following Theorem gives upper and lower bounds on the ground state energy of (3.1) in terms of the ground state energy of the 1D Hamiltonian (1.3). Its proof will be given in Subsections 3.1 and 3.2. A crucial step in the proof of the lower bound will be the use of the 'Dyson Lemma' (see [9,31]), which converts the 'hard' interaction potential $v_{a}$ into a 'soft' one. With this new 'soft' potential perturbation theory can be done, and rigorous bounds are obtained with the use of Temple's inequality (2.61).

Theorem 3.1 (Finite $\boldsymbol{n}$ bounds). Let $E_{\mathrm{D}}^{1 \mathrm{D}}(n, \ell, g)$ and $E_{\mathrm{N}}^{1 \mathrm{D}}(n, \ell, g)$ denote the ground state energy of (1.3) on $L^{2}\left([0, \ell]^{n}\right)$, with Dirichlet and Neumann boundary conditions, respectively, and let $g$ be given by (1.10). Then there is a finite number $C>0$ such that

$$
E_{\mathrm{N}}^{\mathrm{QM}}(n, \ell, r, a)-\frac{n e^{\perp}}{r^{2}} \geq E_{\mathrm{N}}^{1 \mathrm{D}}(n, \ell, g)\left(1-C n\left(\frac{a}{r}\right)^{1 / 8}\left[1+\frac{n r}{\ell}\left(\frac{a}{r}\right)^{1 / 8}\right]\right) .
$$


Moreover,

$$
E_{\mathrm{D}}^{\mathrm{QM}}(n, \ell, r, a)-\frac{n e^{\perp}}{r^{2}} \leq E_{\mathrm{D}}^{1 \mathrm{D}}(n, \ell, g)\left(1+C\left[\left(\frac{n a}{r}\right)^{2}\left(1+\frac{a \ell}{r^{2}}\right)\right]^{1 / 3}\right),
$$

provided the term in square brackets is less than 1.

Let us comment briefly on the error terms in (3.2) and (3.3). As already mentioned, in the proof of Theorem 1.1 we will divide space in the $z$-direction into small boxes of side length $\ell$. The number of particles in each box will be roughly $n \sim N \ell / \bar{L}$, where $\bar{L} \equiv N / \bar{\rho}$ the extension of the system in $z$-direction. The $n$-dependence of the error term in (3.2) restricts us essentially to have a finite particle number $n$, i.e., that $n \sim N \ell / \bar{L} \sim 1$, or $\ell \sim \bar{L} / N$. But for (3.2) to be useful we need $\ell \gtrsim r$, i.e., $r \lesssim \bar{L} / N$, or, in other words, $r$ should be of the order of the mean particle spacing, or smaller. For $r \gg \bar{L} / N, r$ is much bigger than the mean particle spacing, and we have to use a different strategy, similar to the one used in the 3D problem [31, 27]. This will be necessary for the lower bound in Regions 1-3. The result is stated in Theorem 3.2. For it's proof it will be necessary to use the box method also in $\mathbf{x}^{\perp}$-direction, similar to the 3D case considered in [27]. However, one cannot use directly the results from there, one has to be careful to retain uniformity in $r / L$.

Likewise, (3.3) will not be useful for an upper bound in all the Regions $1-5$. The reason is the last term in (3.3), where we want $g \ell \sim g \bar{L} / N \lesssim 1$, which is only fulfilled in Regions 1-4. For Region 5, we use a different upper bound, given in the following Theorem. The proof of Theorem 3.2 will be given in Subsections 3.3 and 3.4.

Theorem 3.2 (Additional energy bounds). With the same notations as in Thm. 3.1,

$$
\begin{aligned}
& E_{\mathrm{N}}^{\mathrm{QM}}(n, \ell, r, a)-\frac{n e^{\perp}}{r^{2}} \geq \\
& \frac{n^{2} g}{2 \ell}\left(1-C\left[n^{-1 / 14}+\left(\frac{n \ell a}{r^{2}}\right)^{1 / 8}+\left(\left[1+\frac{r}{\sqrt{n} \ell}\right]\left(\frac{\sqrt{n} a}{r}\right)^{1 / 4}\right)^{4 / 39}+\frac{n a}{\ell}\right]\right) .
\end{aligned}
$$

Moreover, denoting the range of $v_{a}$ by $R_{0}$,

$$
E_{\mathrm{D}}^{\mathrm{QM}}(n, \ell, r, a)-\frac{n e^{\perp}}{r^{2}} \leq \frac{\pi^{2}}{3} \frac{n^{3}}{\ell^{2}} \frac{(1+1 / n)(1+1 / 2 n)}{\left(1-(n-1) R_{0} / \ell\right)^{2}},
$$

provided $(n-1) R_{0}<\ell$.

Remark. By definition, $R_{0} \sim a$. Eq. (3.4) will be used with $\ell \sim\left(r^{2} \bar{L} / N\right)^{1 / 3}$ and $n \sim N \ell / \bar{L}$. In this case we have, in Regions $1-3, n \ell g \sim a\left(N / r^{2} \bar{L}\right)^{1 / 3} \ll 1$, $n a / \ell \sim a \bar{\rho} \ll 1, \sqrt{n} a / r \sim a\left(N / r^{2} \bar{L}\right)^{1 / 3} \ll 1$ and $r /(\sqrt{n} \ell) \sim 1$.

The following four subsections contain the proofs of Theorems 3.1 and 3.2. Throughout, $C$ denotes a constant independent of the parameters, although the value of different $C$ 's may be different. 


\subsection{Upper bound for Theorem 3.1}

In this subsection we are going to prove (3.3). We use the variational principle. Let $\psi$ denote the ground state of (1.3) with Dirichlet boundary conditions, normalized by $\langle\psi \mid \psi\rangle=1$, and let $G$ and $F$ be given by

$$
G\left(\mathbf{x}_{1}, \ldots, \mathbf{x}_{n}\right)=\psi\left(z_{1}, \ldots, z_{n}\right) \prod_{j=1}^{n} b_{r}\left(\mathbf{x}_{j}^{\perp}\right)
$$

and

$$
F\left(\mathbf{x}_{1}, \ldots, \mathbf{x}_{n}\right)=\prod_{i<j} f\left(\left|\mathbf{x}_{i}-\mathbf{x}_{j}\right|\right) .
$$

Here $f$ is a monotone increasing function, with $0 \leq f \leq 1$ and $f(t)=1$ for $t \geq R$ for some $R \geq R_{0}$. For $t \leq R$ we shall choose $f(t)=f_{0}(t) / f_{0}(R)$, where $f_{0}$ is the solution to the zero-energy scattering equation for $v_{a}[31,27]$. Note that $f_{0}(R)=1-a / R$ for $R \geq R_{0}$, and $f_{0}^{\prime}(t) \leq t^{-1} \min \{1, a / t\}$. We use as a trial wave function for (3.1) the function

$$
\Psi\left(\mathbf{x}_{1}, \ldots, \mathbf{x}_{n}\right)=G\left(\mathbf{x}_{1}, \ldots, \mathbf{x}_{n}\right) F\left(\mathbf{x}_{1}, \ldots, \mathbf{x}_{n}\right) .
$$

Let $\rho_{\psi}^{(2)}$ denote the two-particle density of $\psi$, normalized by $\int \rho_{\psi}^{(2)}\left(z, z^{\prime}\right) d z d z^{\prime}=1$. Since $F$ is 1 whenever no pair of particles is closer together than a distance $R$, we can estimate the norm of $\Psi$ by

$$
\begin{aligned}
& \langle\Psi \mid \Psi\rangle \geq \int G^{2}\left(1-\sum_{i<j} \theta\left(R-\left|\mathbf{x}_{i}-\mathbf{x}_{j}\right|\right)\right) \\
& =1-\frac{n(n-1)}{2} \int \rho_{\psi}^{(2)}\left(z, z^{\prime}\right) b_{r}\left(\mathbf{x}^{\perp}\right)^{2} b_{r}\left(\mathbf{y}^{\perp}\right)^{2} \theta(R-|\mathbf{x}-\mathbf{y}|) d z d z^{\prime} d^{2} \mathbf{x}^{\perp} d^{2} \mathbf{y}^{\perp} \\
& \geq 1-\frac{n(n-1)}{2} \int \rho_{\psi}^{(2)}\left(z, z^{\prime}\right) b_{r}\left(\mathbf{x}^{\perp}\right)^{2} b_{r}\left(\mathbf{y}^{\perp}\right)^{2} \theta\left(R-\left|\mathbf{x}^{\perp}-\mathbf{y}^{\perp}\right|\right) d z d z^{\prime} d^{2} \mathbf{x}^{\perp} d^{2} \mathbf{y}^{\perp} \\
& =1-\frac{n(n-1)}{2} \int b_{r}\left(\mathbf{x}^{\perp}\right)^{2} b_{r}\left(\mathbf{y}^{\perp}\right)^{2} \theta\left(R-\left|\mathbf{x}^{\perp}-\mathbf{y}^{\perp}\right|\right) d^{2} \mathbf{x}^{\perp} d^{2} \mathbf{y}^{\perp} \\
& \geq 1-\frac{n(n-1)}{2} \frac{\pi R^{2}}{r^{2}}\|b\|_{4}^{4},
\end{aligned}
$$

where we used Young's inequality [24] in the last step.

Using

$$
\left\langle\Psi\left|-\Delta_{j}\right| \Psi\right\rangle=-\int F^{2} G \Delta_{j} G+\int G^{2}\left|\nabla_{j} F\right|^{2}
$$

and the Schrödinger equation $H_{n, g} \psi=E_{\mathrm{D}}^{1 \mathrm{D}} \psi$, we can write the expectation value of (3.1) as

$$
\begin{aligned}
\langle\Psi|H| \Psi\rangle= & \left(E_{\mathrm{D}}^{1 \mathrm{D}}+\frac{n}{r^{2}} e^{\perp}\right)\langle\Psi \mid \Psi\rangle-g\left\langle\Psi\left|\sum_{i<j} \delta\left(z_{i}-z_{j}\right)\right| \Psi\right\rangle \\
& +\int G^{2}\left(\sum_{j=1}^{n}\left|\nabla_{j} F\right|^{2}+\sum_{i<j} v_{a}\left(\left|\mathbf{x}_{i}-\mathbf{x}_{j}\right|\right)|F|^{2}\right) .
\end{aligned}
$$


Now, since $0 \leq f \leq 1$ and $f^{\prime} \geq 0$ by assumption, $F^{2} \leq f\left(\left|\mathbf{x}_{i}-\mathbf{x}_{j}\right|\right)^{2}$, and

$$
\sum_{j=1}^{n}\left|\nabla_{j} F\right|^{2} \leq 2 \sum_{i<j} f^{\prime}\left(\left|\mathbf{x}_{i}-\mathbf{x}_{j}\right|\right)^{2}+4 \sum_{k<i<j} f^{\prime}\left(\left|\mathbf{x}_{k}-\mathbf{x}_{i}\right|\right) f^{\prime}\left(\left|\mathbf{x}_{k}-\mathbf{x}_{j}\right|\right) .
$$

Consider the first term on the right side of (3.12), together with the last term in (3.11). These terms are bounded above by

$$
\begin{aligned}
& 2 \sum_{i<j} \int G^{2}\left(f^{\prime}\left(\left|\mathbf{x}_{i}-\mathbf{x}_{j}\right|\right)^{2}+\frac{1}{2} v_{a}\left(\left|\mathbf{x}_{i}-\mathbf{x}_{j}\right|\right) f\left(\left|\mathbf{x}_{i}-\mathbf{x}_{j}\right|\right)^{2}\right) \\
& =n(n-1) \int b_{r}\left(\mathbf{x}^{\perp}\right)^{2} b_{r}\left(\mathbf{y}^{\perp}\right)^{2} \rho_{\psi}^{(2)}\left(z, z^{\prime}\right)\left(f^{\prime}(|\mathbf{x}-\mathbf{y}|)^{2}+\frac{1}{2} v_{a}(|\mathbf{x}-\mathbf{y}|) f(|\mathbf{x}-\mathbf{y}|)^{2}\right) .
\end{aligned}
$$

Let

$$
h(z)=\int\left(f^{\prime}(|\mathbf{x}|)^{2}+\frac{1}{2} v_{a}(|\mathbf{x}|) f(|\mathbf{x}|)^{2}\right) d^{2} \mathbf{x}^{\perp} .
$$

Note that $h(z)=0$ for $|z| \geq R$, and $\int h(z) d z=4 \pi a(1-a / R)^{-1}$. Using Young's inequality for the integration over the $\perp$-variables, we get

$$
(3.13) \leq \frac{n(n-1)}{r^{2}}\|b\|_{4}^{4} \int_{\mathbb{R}^{2}} \rho_{\psi}^{(2)}\left(z, z^{\prime}\right) h\left(z-z^{\prime}\right) d z d z^{\prime} .
$$

Consider now the contribution from the last term in (3.12). We can write

$$
\begin{aligned}
& 4 \sum_{k<i<j} \int G^{2} f^{\prime}\left(\left|\mathbf{x}_{k}-\mathbf{x}_{i}\right|\right) f^{\prime}\left(\left|\mathbf{x}_{k}-\mathbf{x}_{j}\right|\right)=\frac{2}{3} n(n-1)(n-2) \\
& \cdot \int f^{\prime}\left(\left|\mathbf{x}_{1}-\mathbf{x}_{2}\right|\right) f^{\prime}\left(\left|\mathbf{x}_{2}-\mathbf{x}_{3}\right|\right) b_{r}\left(\mathbf{x}_{1}^{\perp}\right)^{2} b_{r}\left(\mathbf{x}_{2}^{\perp}\right)^{2} b_{r}\left(\mathbf{x}_{3}^{\perp}\right)^{2} \rho_{\psi}^{(3)}\left(z_{1}, z_{2}, z_{3}\right) d^{3} \mathbf{x}_{1} d^{3} \mathbf{x}_{2} d^{3} \mathbf{x}_{3}
\end{aligned}
$$

where $\rho_{\psi}^{(3)}$ denotes the three-particle density of $\psi$, normalized by 1 . Let

$$
k(z)=\int f^{\prime}(|\mathbf{x}|) d^{2} \mathbf{x}^{\perp},
$$

which is supported in $[-R, R]$. For the integration over $\mathbf{x}_{1}^{\perp}$ we use

$$
\int f^{\prime}\left(\left|\mathbf{x}_{1}-\mathbf{x}_{2}\right|\right) b_{r}\left(\mathbf{x}_{1}^{\perp}\right)^{2} d^{2} \mathbf{x}_{1}^{\perp} \leq \frac{\|b\|_{\infty}^{2}}{r^{2}} k\left(z_{1}-z_{2}\right) \leq \frac{\|b\|_{\infty}^{2}}{r^{2}}\|k\|_{\infty}
$$

For the remaining integrations, we proceed as in (3.15) to obtain

$$
(3.16) \leq \frac{2}{3} n(n-1)(n-2) \frac{\|b\|_{\infty}^{2}}{r^{2}} \frac{\|b\|_{4}^{4}}{r^{2}}\|k\|_{\infty} \int_{\mathbb{R}^{2}} \rho_{\psi}^{(2)}\left(z, z^{\prime}\right) k\left(z-z^{\prime}\right) d z d z^{\prime} .
$$


Now, for any $\phi \in H^{1}(\mathbb{R})$,

$$
\begin{aligned}
\left.|| \phi(z)\right|^{2}-\left|\phi\left(z^{\prime}\right)\right|^{2} \mid & =\left|\int_{z^{\prime}}^{z} \frac{d\left|\phi\left(z^{\prime}\right)\right|^{2}}{d z^{\prime}} d z^{\prime}\right| \leq 2\|\phi\|_{\infty} \int_{z^{\prime}}^{z}\left|\frac{d \phi\left(z^{\prime}\right)}{d z^{\prime}}\right| d z^{\prime} \\
& \leq 2\left|z-z^{\prime}\right|^{1 / 2}\left(\int_{\mathbb{R}}|\phi|^{2}\right)^{1 / 4}\left(\int_{\mathbb{R}}\left|\frac{d \phi}{d z}\right|^{2}\right)^{3 / 4}
\end{aligned}
$$

where we used $\|\phi\|_{\infty}^{2} \leq\|d \phi / d z\|_{2}\|\phi\|_{2}$. Applying this to $\rho_{\psi}^{(2)}\left(z, z^{\prime}\right)$, considered as a function of $z$ only, and using the fact that the support of $h$ is contained in $[-R, R]$, we therefore get

$$
\begin{aligned}
& \int_{\mathbb{R}^{2}} \rho_{\psi}^{(2)}\left(z, z^{\prime}\right) h\left(z-z^{\prime}\right) d z d z^{\prime}-\int_{\mathbb{R}} h(z) d z \int \rho_{\psi}^{(2)}(z, z) d z \\
& \leq 2 R^{1 / 2} \int_{\mathbb{R}} h(z) d z \int\left|\partial_{z} \sqrt{\rho_{\psi}^{(2)}\left(z, z^{\prime}\right)}\right|^{2} d z d z^{\prime} \leq 2 R^{1 / 2} \int_{\mathbb{R}} h(z) d z\left\langle\psi\left|-\frac{d^{2}}{d z_{1}^{2}}\right| \psi\right\rangle^{3 / 4}
\end{aligned}
$$

where we used Schwarz' inequality, the normalization of $\rho_{\psi}^{(2)}$ and the symmetry of $\psi$. The same argument is used for (3.19) with $h$ replaced by $k$. Now $\int h(z) d z=$ $4 \pi a(1-a / R)^{-1}$, and

$$
\begin{aligned}
\|k\|_{\infty} & \leq \frac{2 \pi a}{1-a / R}(1+\ln (R / a)) \\
\int_{\mathbb{R}} k(z) d z & \leq \frac{2 \pi a R}{1-a / R}\left(1-\frac{a}{2 R}\right) .
\end{aligned}
$$

Therefore

$$
(3.15)+(3.19) \leq \frac{n(n-1)}{2} g \frac{1+K}{1-a / R}\left[\int \rho_{\psi}^{(2)}(z, z) d z+2 R^{1 / 2}\left\langle\psi\left|-\frac{d^{2}}{d z_{1}^{2}}\right| \psi\right\rangle^{3 / 4}\right]
$$

where we denoted

$$
K=\frac{2 \pi}{3}(n-2) \frac{a}{R} \frac{1+\ln (R / a)}{1-a / R}\left(\frac{R}{r}\right)^{2}\|b\|_{\infty}^{2} .
$$

It remains to bound the second term in (3.11). We use again the fact that $F$ is equal to 1 as long as the particles are not within a distance $R$. We obtain

$$
\begin{aligned}
& \left\langle\Psi\left|\sum_{i<j} \delta\left(z_{i}-z_{j}\right)\right| \Psi\right\rangle \\
& \geq \frac{n(n-1)}{2} \int \rho_{\psi}^{(2)}(z, z) d z \int b_{r}\left(\mathbf{x}^{\perp}\right)^{2} b_{r}\left(\mathbf{y}^{\perp}\right)^{2}\left(1-\theta\left(R-\left|\mathbf{x}^{\perp}-\mathbf{y}^{\perp}\right|\right)\right) \\
& \geq \frac{n(n-1)}{2} \int \rho_{\psi}^{(2)}(z, z) d z\left(1-\frac{\pi R^{2}}{r^{2}}\|b\|_{4}^{4}\right) .
\end{aligned}
$$


Putting together the bounds (3.9), (3.23) and (3.25), and using the fact that

$g \frac{1}{2} n(n-1) \int \rho_{\psi}^{(2)}(z, z) d z \leq E_{\mathrm{D}}^{1 \mathrm{D}}$ and $\left\langle\psi\left|-d^{2} / d z_{1}^{2}\right| \psi\right\rangle \leq E_{\mathrm{D}}^{1 \mathrm{D}} / n$, we obtain the upper bound

$$
\begin{aligned}
& \frac{\langle\Psi|H| \Psi\rangle}{\langle\Psi \mid \Psi\rangle}-\frac{n e^{\perp}}{r^{2}} \leq E_{\mathrm{D}}^{1 \mathrm{D}}(n, \ell, g)\left[1-\frac{n(n-1)}{2} \frac{\pi R^{2}}{r^{2}}\|b\|_{4}^{4}\right]^{-1} \\
& \cdot\left(1+\frac{a / R+K}{(1-a / R)}+\frac{\pi R^{2}}{r^{2}}\|b\|_{4}^{4}+g(n-1) R^{1 / 2}\left(\frac{n}{E_{\mathrm{D}}^{1 \mathrm{D}}}\right)^{1 / 4} \frac{1+K}{1-a / R}\right)
\end{aligned}
$$

provided the term in square brackets is positive. We now use $E_{\mathrm{D}}^{1 \mathrm{D}} / n \geq(\pi / \ell)^{2}$, and choose

$$
R^{3}=\frac{a r^{2}}{n^{2}(1+g \ell)}
$$

This gives (under the assumption $(n a / r)^{2}(1+g \ell) \leq 1$ )

$$
E_{\mathrm{D}}^{\mathrm{QM}}(n, \ell, r, a)-\frac{n e^{\perp}}{r^{2}} \leq E_{\mathrm{D}}^{1 \mathrm{D}}(n, \ell, g)\left(1+C\left(\frac{n a}{r}\right)^{2 / 3}(1+g \ell)^{1 / 3}\right)
$$

for some constant $C>0$.

\subsection{Lower bound for Theorem 3.1}

We are now going to prove (3.2). We write a general wave function $\Psi$ as

$$
\Psi\left(\mathbf{x}_{1}, \ldots, \mathbf{x}_{n}\right)=f\left(\mathbf{x}_{1}, \ldots, \mathbf{x}_{n}\right) \prod_{k=1}^{n} b_{r}\left(\mathbf{x}_{k}^{\perp}\right)
$$

which can always be done, since $b_{r}$ is a strictly positive function. Partial integration gives

$$
\langle\Psi|H| \Psi\rangle=\frac{n e^{\perp}}{r^{2}}+\sum_{i=1}^{n} \int\left[\left|\nabla_{i} f\right|^{2}+\frac{1}{2} \sum_{j, j \neq i} v_{a}\left(\left|\mathbf{x}_{i}-\mathbf{x}_{j}\right|\right)|f|^{2}\right] \prod_{k=1}^{n} b_{r}\left(\mathbf{x}_{k}^{\perp}\right)^{2} d^{3} \mathbf{x}_{k}
$$

Choose some $R>R_{0}$, fix $i$ and $\mathbf{x}_{j}, j \neq i$, and consider the Voronoi cell $\Omega_{j}$ around particle $j$, i.e.,

$$
\Omega_{j}=\left\{\mathbf{x}:\left|\mathbf{x}-\mathbf{x}_{j}\right| \leq\left|\mathbf{x}-\mathbf{x}_{k}\right| \text { for all } k \neq j\right\} .
$$

Denote by $\mathcal{B}_{j}$ the ball of radius $R$ around $\mathbf{x}_{j}$. We can estimate

$$
\begin{aligned}
& \int_{\Omega_{j} \cap \mathcal{B}_{j}} b_{r}\left(\mathbf{x}_{i}^{\perp}\right)^{2}\left(\left|\nabla_{i} f\right|^{2}+\frac{1}{2} v_{a}\left(\left|\mathbf{x}_{i}-\mathbf{x}_{j}\right|\right)|f|^{2}\right) d^{3} \mathbf{x}_{i} \\
& \geq \min _{\mathbf{x} \in \mathcal{B}_{j}} b_{r}\left(\mathbf{x}^{\perp}\right)^{2} a \int_{\Omega_{j} \cap \mathcal{B}_{j}} U\left(\left|\mathbf{x}_{i}-\mathbf{x}_{j}\right|\right)|f|^{2} \\
& \geq \frac{\min _{\mathbf{x} \in \mathcal{B}_{j}} b_{r}\left(\mathbf{x}^{\perp}\right)^{2}}{\max _{\mathbf{x} \in \mathcal{B}_{j}} b_{r}\left(\mathbf{x}^{\perp}\right)^{2}} a \int_{\Omega_{j} \cap \mathcal{B}_{j}} b_{r}\left(\mathbf{x}_{i}^{\perp}\right)^{2} U\left(\left|\mathbf{x}_{i}-\mathbf{x}_{j}\right|\right)|f|^{2}
\end{aligned}
$$


where we used Lemma 1 of [31], and

$$
U(r)= \begin{cases}3\left(R^{3}-R_{0}^{3}\right)^{-1} & \text { for } R_{0} \leq r \leq R \\ 0 & \text { otherwise }\end{cases}
$$

For some $\delta>0$ define $\mathcal{B}_{\delta} \subset \mathbb{R}^{2}$ by

$$
\mathcal{B}_{\delta}=\left\{\mathbf{x}^{\perp} \in \mathbb{R}^{2}: b\left(\mathbf{x}^{\perp}\right)^{2} \geq \delta\right\} .
$$

Estimating $\max _{\mathbf{x} \in \mathcal{B}_{j}} b_{r}\left(\mathbf{x}^{\perp}\right)^{2} \leq \min _{\mathbf{x} \in \mathcal{B}_{j}} b_{r}\left(\mathbf{x}^{\perp}\right)^{2}+2\left(R / r^{3}\right)\left\|\nabla b^{2}\right\|_{\infty}$, we obtain

$$
\frac{\min _{\mathbf{x} \in \mathcal{B}_{j}} b_{r}\left(\mathbf{x}^{\perp}\right)^{2}}{\max _{\mathbf{x} \in \mathcal{B}_{j}} b_{r}\left(\mathbf{x}^{\perp}\right)^{2}} \geq \chi_{\mathcal{B}_{\delta}}\left(\mathbf{x}_{j}^{\perp} / r\right)\left(1-2 \frac{R}{r} \frac{\left\|\nabla b^{2}\right\|_{\infty}}{\delta}\right) .
$$

(For a proof that $\nabla b^{2}$ is a bounded function, see the proof of Lemma 1 in the Appendix). Here $\chi_{\mathcal{B}_{\delta}}$ denotes the characteristic function of $\mathcal{B}_{\delta}$. Denoting $k(i)$ the nearest neighbor to particle $i$, we conclude that, for $0 \leq \epsilon \leq 1$,

$$
\begin{gathered}
\sum_{i=1}^{n} \int\left[\left|\nabla_{i} f\right|^{2}+\frac{1}{2} \sum_{j, j \neq i} v_{a}\left(\left|\mathbf{x}_{i}-\mathbf{x}_{j}\right|\right)|f|^{2}\right] \prod_{k=1}^{n} b_{r}\left(\mathbf{x}_{k}^{\perp}\right)^{2} d^{3} \mathbf{x}_{k} \\
\geq \sum_{i=1}^{n} \int\left[\epsilon\left|\nabla_{i} f\right|^{2}+(1-\epsilon)\left|\nabla_{i} f\right|^{2} \chi_{\min _{k}\left|z_{i}-z_{k}\right| \geq R}\left(z_{i}\right)\right. \\
\left.+a^{\prime} U\left(\left|\mathbf{x}_{i}-\mathbf{x}_{k(i)}\right|\right) \chi_{\mathcal{B}_{\delta}}\left(\mathbf{x}_{k(i)}^{\perp} / r\right)|f|^{2}\right] \prod_{k=1}^{n} b_{r}\left(\mathbf{x}_{k}^{\perp}\right)^{2} d^{3} \mathbf{x}_{k}
\end{gathered}
$$

where $a^{\prime}=a(1-\epsilon)\left(1-2 R\left\|\nabla b^{2}\right\|_{\infty} / r \delta\right)$.

Define $F\left(z_{1}, \ldots, z_{n}\right) \geq 0$ by

$$
\left|F\left(z_{1}, \ldots, z_{n}\right)\right|^{2}=\int\left|f\left(\mathbf{x}_{1}, \ldots, \mathbf{x}_{n}\right)\right|^{2} \prod_{k=1}^{n} b_{r}\left(\mathbf{x}_{k}^{\perp}\right)^{2} d^{2} \mathbf{x}_{k}^{\perp} .
$$

Neglecting the kinetic energy in $\perp$-direction in the second term in (3.36) and using the Schwarz inequality to bound the longitudinal kinetic energy of $f$ by the one of $F$, we get the estimate

$$
\begin{aligned}
& \langle\Psi|H| \Psi\rangle-\frac{n e^{\perp}}{r^{2}} \geq \\
& \sum_{i=1}^{n} \int\left[\epsilon\left|\partial_{i} F\right|^{2}+(1-\epsilon)\left|\partial_{i} F\right|^{2} \chi_{\min _{k}}\left|z_{i}-z_{k}\right| \geq R\right. \\
& \left.+\sum_{i=1}^{n} \int[\epsilon)\right] \prod_{k=1}^{n} d z_{k} \\
& \left.+\left.\nabla_{i}^{\perp} f\right|^{2}+a^{\prime} U\left(\left|\mathbf{x}_{i}-\mathbf{x}_{k(i)}\right|\right) \chi_{\mathcal{B}_{\delta}}\left(\mathbf{x}_{k(i)}^{\perp} / r\right)|f|^{2}\right] \prod_{k=1}^{n} b_{r}\left(\mathbf{x}_{k}^{\perp}\right)^{2} d^{3} \mathbf{x}_{k}
\end{aligned}
$$


where $\partial_{j}=d / d z_{j}$, and $\nabla^{\perp}$ denotes the gradient in $\perp$-direction. We now investigate the last term in (3.38). Consider, for fixed $z_{1}, \ldots, z_{n}$, the expression

$$
\sum_{i=1}^{n} \int\left[\epsilon\left|\nabla_{i}^{\perp} f\right|^{2}+a^{\prime} U\left(\left|\mathbf{x}_{i}-\mathbf{x}_{k(i)}\right|\right) \chi_{\mathcal{B}_{\delta}}\left(\mathbf{x}_{k(i)}^{\perp} / r\right)|f|^{2}\right] \prod_{k=1}^{n} b_{r}\left(\mathbf{x}_{k}^{\perp}\right)^{2} d^{2} \mathbf{x}_{k}^{\perp} .
$$

To estimate this term from below, we use Temple's inequality, as in [31]. Let $\widetilde{e}^{\perp}$ denote the gap above zero in the spectrum of $-\Delta^{\perp}+V^{\perp}-e^{\perp}$, i.e., the lowest non-zero eigenvalue. By scaling, $\widetilde{e}^{\perp} / r^{2}$ is the gap in the spectrum of $-\Delta^{\perp}+V_{r}^{\perp}-e^{\perp} / r^{2}$. Note that under the transformation $\phi \mapsto b_{r}^{-1} \phi$ this latter operator is unitarily equivalent to $\nabla^{\perp *} \cdot \nabla^{\perp}$ as an operator on $L^{2}\left(\mathbb{R}^{2}, b_{r}\left(\mathbf{x}^{\perp}\right)^{2} d^{2} \mathbf{x}^{\perp}\right)$, as considered in (3.39). Hence also this operator has $\widetilde{e}^{\perp} / r^{2}$ as its energy gap. Denote

$$
\left\langle U^{k}\right\rangle=\int\left(\sum_{i=1}^{n} U\left(\left|\mathbf{x}_{i}-\mathbf{x}_{k(i)}\right|\right) \chi_{\mathcal{B}_{\delta}}\left(\mathbf{x}_{k(i)}^{\perp} / r\right)\right)^{k} \prod_{k=1}^{n} b_{r}\left(\mathbf{x}_{k}^{\perp}\right)^{2} d^{2} \mathbf{x}_{k}^{\perp} .
$$

Temple's inequality (2.61) implies (under the assumption that the denominator in the last term is positive)

$$
(3.39) \geq|F|^{2} a^{\prime}\langle U\rangle\left(1-a^{\prime} \frac{\left\langle U^{2}\right\rangle}{\langle U\rangle} \frac{1}{\epsilon \widetilde{e}^{\perp} / r^{2}-a^{\prime}\langle U\rangle}\right) .
$$

Now, using (3.33) and Schwarz' inequality, $\left\langle U^{2}\right\rangle \leq 3 n\left(R^{3}-R_{0}^{3}\right)^{-1}\langle U\rangle$, and

$$
\begin{aligned}
\langle U\rangle & \leq n(n-1) \int U(|\mathbf{x}-\mathbf{y}|) b_{r}\left(\mathbf{x}^{\perp}\right)^{2} b_{r}\left(\mathbf{y}^{\perp}\right)^{2} d^{2} \mathbf{x}^{\perp} d^{2} \mathbf{y}^{\perp} \\
& \leq n(n-1) \frac{\|b\|_{4}^{4}}{r^{2}} \int U(|\mathbf{x}|) d^{2} \mathbf{x}^{\perp} \leq n(n-1) \frac{\|b\|_{4}^{4}}{r^{2}} \frac{3 \pi R^{2}}{R^{3}-R_{0}^{3}} .
\end{aligned}
$$

Using this and $a^{\prime} \leq a$ in the error term, we obtain

$$
(3.41) \geq|F|^{2} a^{\prime \prime}\langle U\rangle,
$$

where

$$
a^{\prime \prime}=a^{\prime}\left(1-\frac{3 n}{\epsilon \widetilde{e}^{\perp}} \frac{a r^{2}}{R^{3}} \frac{1}{1-\left(R_{0} / R\right)^{3}}\left[1-\frac{n^{2}}{\epsilon \widetilde{e}^{\perp}} \frac{a}{R} 3 \pi\|b\|_{4}^{4} \frac{1}{1-\left(R_{0} / R\right)^{3}}\right]^{-1}\right),
$$

with the understanding that the term in square brackets is positive. Now let

$$
d\left(z-z^{\prime}\right)=\int_{\mathbb{R}^{4}} b_{r}\left(\mathbf{x}^{\perp}\right)^{2} b_{r}\left(\mathbf{y}^{\perp}\right)^{2} U(|\mathbf{x}-\mathbf{y}|) \chi_{\mathcal{B}_{\delta}}\left(\mathbf{y}^{\perp} / r\right) d^{2} \mathbf{x}^{\perp} d^{2} \mathbf{y}^{\perp} .
$$

Note that $d(z)=0$ if $|z| \geq R$. We estimate $\langle U\rangle$ from below by

$$
\begin{aligned}
\langle U\rangle & \geq \sum_{i \neq j} \int U\left(\left|\mathbf{x}_{i}-\mathbf{x}_{j}\right|\right) \chi_{\mathcal{B}_{\delta}}\left(\mathbf{x}_{j}^{\perp} / r\right) \prod_{k, k \neq i, j} \theta\left(\left|\mathbf{x}_{k}-\mathbf{x}_{i}\right|-R\right) \prod_{l=1}^{n} b_{r}\left(\mathbf{x}_{l}^{\perp}\right)^{2} d^{2} \mathbf{x}_{l}^{\perp} \\
& \geq \sum_{i \neq j} \int U\left(\left|\mathbf{x}_{i}-\mathbf{x}_{j}\right|\right) \chi_{\mathcal{B}_{\delta}}\left(\mathbf{x}_{j}^{\perp} / r\right)\left(1-\sum_{k, k \neq i, j} \theta\left(R-\left|\mathbf{x}_{k}-\mathbf{x}_{i}\right|\right)\right) \prod_{l=1}^{n} b_{r}\left(\mathbf{x}_{l}^{\perp}\right)^{2} d^{2} \mathbf{x}_{l}^{\perp} \\
& \geq \sum_{i \neq j} d\left(z_{i}-z_{j}\right)\left(1-(n-2) \frac{\pi R^{2}}{r^{2}}\|b\|_{\infty}^{2}\right) .
\end{aligned}
$$


Let

$$
a^{\prime \prime \prime}=a^{\prime \prime}\left(1-(n-2) \frac{\pi R^{2}}{r^{2}}\|b\|_{\infty}^{2}\right)
$$

and denote $g^{\prime}=2 a^{\prime \prime \prime} \int_{\mathbb{R}} d(z) d z$. Note that since $\left|b\left(\mathbf{x}^{\perp}\right)^{2}-b\left(\mathbf{y}^{\perp}\right)^{2}\right| \leq R\left\|\nabla b^{2}\right\|_{\infty}$ for $\left|\mathbf{x}^{\perp}-\mathbf{y}^{\perp}\right| \leq R$

$$
\begin{aligned}
\int_{\mathbb{R}} d(z) d z & \geq \frac{4 \pi}{r^{2}}\left(\int_{\mathcal{B}_{\delta}} b\left(\mathbf{x}^{\perp}\right)^{4} d^{2} \mathbf{x}^{\perp}-R\left\|\nabla b^{2}\right\|_{\infty} / r\right) \\
& \geq \frac{4 \pi}{r^{2}}\left(\|b\|_{4}^{4}-\delta-R\left\|\nabla b^{2}\right\|_{\infty} / r\right) .
\end{aligned}
$$

We write

$$
\begin{aligned}
& \sum_{i=1}^{n} \int\left[\epsilon\left|\partial_{i} F\right|^{2}+a^{\prime \prime \prime} \sum_{j, j \neq i} d\left(z_{i}-z_{j}\right)|F|^{2}\right] \prod_{k=1}^{n} d z_{k} \\
& =\sum_{i \neq j} \int\left[\frac{\epsilon}{n-1}\left|\partial_{i} F\right|^{2}+a^{\prime \prime \prime} d\left(z_{i}-z_{j}\right)|F|^{2}\right] \prod_{k=1}^{n} d z_{k} .
\end{aligned}
$$

Now consider, for fixed $z_{j}, j \neq i$, the expression

$$
\int\left[\frac{\epsilon}{n-1}\left|\partial_{i} F\right|^{2}+a^{\prime \prime \prime} d\left(z_{i}-z_{j}\right)|F|^{2}\right] d z_{i}
$$

We claim that

$$
(3.50) \geq \frac{1}{2} g^{\prime} \max _{\left|z_{i}-z_{j}\right| \leq R}|F|^{2} \chi_{[R, \ell-R]}\left(z_{j}\right)\left(1-\left(\frac{2(n-1)}{\epsilon} g^{\prime} R\right)^{1 / 2}\right) .
$$

Assume that (3.51) is false. Estimating, for any $H^{1}([0, \ell])$-function $\phi$,

$$
|\phi(z)|^{2}-\max _{\left|z-z_{0}\right| \leq R}|\phi(z)|^{2} \geq-\left.\left|\int_{z}^{z_{0}} \partial\right| \phi\right|^{2}\left|\geq-2 R^{1 / 2} \max _{\left|z-z_{0}\right| \leq R}\right| \phi(z) \mid\left(\int_{0}^{\ell}|\partial \phi|^{2}\right)^{1 / 2}
$$

and applying this estimate to $F$ (considered only as a function of $z_{i}$ ), we obtain, using $\epsilon \int\left|\partial_{i} F\right|^{2} \leq \frac{1}{2}(n-1) g^{\prime} \max _{\left|z_{i}-z_{j}\right| \leq R}|F|^{2}$ by assumption,

$$
\begin{aligned}
& a^{\prime \prime \prime} \int d\left(z_{i}-z_{j}\right)|F|^{2} d z_{i} \\
& \geq a^{\prime \prime \prime} \int d\left(z-z_{j}\right) d z \max _{\left|z_{i}-z_{j}\right| \leq R}|F|^{2}\left(1-\left(\frac{2}{\epsilon}(n-1) g^{\prime} R\right)^{1 / 2}\right) \\
& \geq \frac{1}{2} g^{\prime} \chi_{[R, \ell-R]}\left(z_{j}\right) \max _{\left|z_{i}-z_{j}\right| \leq R}|F|^{2}\left(1-\left(\frac{2}{\epsilon}(n-1) g^{\prime} R\right)^{1 / 2}\right),
\end{aligned}
$$

contradicting our assumption. This proves (3.51). 
Putting everything together, we thus obtain

$$
\begin{aligned}
\langle\Psi|H| \Psi\rangle-\frac{n e^{\perp}}{r^{2}} \geq & \sum_{i=1}^{n} \int\left[(1-\epsilon)\left|\partial_{i} F\right|^{2} \chi_{\min _{k}\left|z_{i}-z_{k}\right| \geq R}\left(z_{i}\right)\right] \prod_{k=1}^{n} d z_{k} \\
& +\sum_{i \neq j} \frac{1}{2} g^{\prime \prime} \int_{\left|z_{i}-z_{j}\right| \leq R}|F|^{2} \chi_{[R, \ell-R]}\left(z_{j}\right) \prod_{k, k \neq i} d z_{k},
\end{aligned}
$$

where

$$
g^{\prime \prime}=g^{\prime}\left(1-\left(\frac{2}{\epsilon}(n-1) g^{\prime} R\right)^{1 / 2}\right) .
$$

Assume that $(n+1) R<\ell$. Given an $F$ with $\int|F|^{2} d z_{1} \cdots d z_{n}=1$, define, for $0 \leq z_{1} \leq z_{2} \leq \cdots \leq z_{n} \leq \ell-(n+1) R$,

$$
\psi\left(z_{1}, \ldots, z_{n}\right)=F\left(z_{1}+R, z_{2}+2 R, z_{3}+3 R, \ldots, z_{n}+n R\right),
$$

and extend the function to all of $[0, \ell-(n+1) R]^{n}$ by symmetry. A simple calculation shows that, for

$$
H^{\prime}=(1-\epsilon) \sum_{i=1}^{n}-\partial_{i}^{2}+g^{\prime \prime} \sum_{i<j} \delta\left(z_{i}-z_{j}\right)
$$

on $L^{2}\left([0, \ell-(n+1) R]^{n}\right)$,

$$
\begin{aligned}
(3.54) \geq\left\langle\psi\left|H^{\prime}\right| \psi\right\rangle & \geq(1-\epsilon) E_{\mathrm{N}}^{1 \mathrm{D}}\left(n, \ell-(n+1) R, g^{\prime \prime}\right)\langle\psi \mid \psi\rangle \\
& \geq(1-\epsilon) E_{\mathrm{N}}^{1 \mathrm{D}}\left(n, \ell, g^{\prime \prime}\right)\langle\psi \mid \psi\rangle .
\end{aligned}
$$

Here $\left\langle\psi\left|H^{\prime}\right| \psi\right\rangle$ is interpreted in the quadratic from sense, since $\psi$ does not necessarily fulfill Neumann boundary conditions. Since these give the lowest energy for the quadratic form, (3.58) is valid anyway.

It remains to estimate $\langle\psi \mid \psi\rangle$ for the $F$ that is related to the true ground state $\Psi$ by (3.37). We have

$$
\begin{aligned}
\langle\psi \mid \psi\rangle & =\int|F|^{2} \prod_{k=1}^{n} \chi_{[R, \ell-R]}\left(z_{k}\right) \prod_{i<j} \theta\left(\left|z_{i}-z_{j}\right|-R\right) \\
& \geq 1-\int|F|^{2}\left[\sum_{k=1}^{n}\left(1-\chi_{[R, \ell-R]}\left(z_{k}\right)\right)+\sum_{i<j} \theta\left(R-\left|z_{i}-z_{j}\right|\right) \chi_{[R, \ell-R]}\left(z_{j}\right)\right] .
\end{aligned}
$$

To bound the second term on the right side of (3.59), we use

$$
\begin{aligned}
\sum_{i<j} \int|F|^{2} \theta\left(R-\left|z_{i}-z_{j}\right|\right) \chi_{[R, \ell-R]}\left(z_{j}\right) & \leq R \sum_{i \neq j} \int \max _{\left|z_{i}-z_{j}\right| \leq R}|F|^{2} \chi_{[R, \ell-R]}\left(z_{j}\right) \\
& \leq \frac{2 R}{g^{\prime \prime}}\left(E_{\mathrm{N}}^{\mathrm{QM}}(N, \ell, r, a)-\frac{n e^{\perp}}{r^{2}}\right),(3.66
\end{aligned}
$$


where the last inequality follows from (3.54). For the other term, we use the simple fact that, for any function $\phi \in H^{1}([0, \ell])$, and for $0 \leq R \leq \ell$,

$$
\int_{0}^{R} \phi(z) d z-\frac{R}{\ell} \int_{0}^{\ell} \phi(z) d z=\int_{0}^{L} \phi^{\prime}(z) f_{R, \ell}(z) d z
$$

with $f_{R, \ell}(z)=z R / \ell-\min \{z, R\}$. Note that $\left|f_{R, \ell}(z)\right| \leq R$. Applying this to $F^{2}$ and using Schwarz' inequality we get the estimate

$$
\begin{aligned}
\int|F|^{2} \sum_{k=1}^{n}\left(1-\chi_{[R, \ell-R]}\left(z_{k}\right)\right) & \leq 2 n \frac{R}{\ell}+4 n R\left(\frac{1}{n}\left\langle F\left|\sum_{i}-\partial_{i}^{2}\right| F\right\rangle\right)^{1 / 2} \\
& \leq 2 n \frac{R}{\ell}+4 n R\left(\frac{1}{n} E_{\mathrm{N}}^{\mathrm{QM}}(n, \ell, r, a)-\frac{e^{\perp}}{r^{2}}\right)^{1 / 2} .
\end{aligned}
$$

Denoting $A \equiv E_{\mathrm{N}}^{\mathrm{QM}}(n, \ell, r, a)-n e^{\perp} / r^{2}$, we thus have

$$
A \geq E_{\mathrm{N}}^{1 \mathrm{D}}\left(n, \ell, g^{\prime \prime}\right)\left(1-\frac{2 R}{g^{\prime \prime}} A-2 n \frac{R}{\ell}-4 \sqrt{n} R A^{1 / 2}\right)
$$

which implies

$$
A \geq \frac{E_{\mathrm{N}}^{1 \mathrm{D}}\left(n, \ell, g^{\prime \prime}\right)}{1+2 R E_{\mathrm{N}}^{1 \mathrm{D}}\left(n, \ell, g^{\prime \prime}\right) / g^{\prime \prime}}\left(1-2 n \frac{R}{\ell}-2 R \sqrt{n E_{\mathrm{N}}^{1 \mathrm{D}}\left(n, \ell, g^{\prime \prime}\right)}\right) .
$$

We now use the simple upper bounds

$$
E_{\mathrm{N}}^{1 \mathrm{D}}\left(n, \ell, g^{\prime \prime}\right) \leq \frac{n(n-1)}{2 \ell} g^{\prime \prime} \quad \text { and } \quad E_{\mathrm{N}}^{1 \mathrm{D}}\left(n, \ell, g^{\prime \prime}\right) \leq \frac{\pi^{2}}{3} \frac{n^{3}}{\ell^{2}}
$$

which follow from a constant and a free-fermion trial wave function, respectively. Moreover, by concavity of $E_{\mathrm{N}}^{1 \mathrm{D}}$ in $g, E_{\mathrm{N}}^{1 \mathrm{D}}\left(n, \ell, g^{\prime \prime}\right) \geq E_{\mathrm{N}}^{1 \mathrm{D}}(n, \ell, g) g^{\prime \prime} / g$ for $g^{\prime \prime} \leq g$, and therefore

$$
E_{\mathrm{N}}^{\mathrm{QM}}(n, \ell, r, a)-\frac{n e^{\perp}}{r^{2}} \geq E_{\mathrm{N}}^{1 \mathrm{D}}(n, \ell, g) \frac{g^{\prime \prime}}{g}\left(1-\frac{R}{\ell}\left[n(n+1)+\frac{2 \pi}{\sqrt{3}} n^{2}\right]\right) .
$$

We now choose

$$
R=r\left(\frac{a}{r}\right)^{1 / 4}, \quad \epsilon=\left(\frac{a}{r}\right)^{1 / 8}, \quad \delta=\left(\frac{a}{r}\right)^{1 / 8}
$$

and obtain

$$
E_{\mathrm{N}}^{\mathrm{QM}}(n, \ell, r, a)-\frac{n e^{\perp}}{r^{2}} \geq E_{\mathrm{N}}^{1 \mathrm{D}}(n, \ell, g)\left(1-C n\left(\frac{a}{r}\right)^{1 / 8}\left[1+\frac{n r}{\ell}\left(\frac{a}{r}\right)^{1 / 8}\right]\right)
$$

for some constant $C>0$. 


\subsection{Upper bound for the Girardeau-Tonks regime}

We use as a trial function

$$
\Psi\left(\mathbf{x}_{1}, \ldots, \mathbf{x}_{n}\right)=\psi\left(z_{1}, \ldots, z_{n}\right) \prod_{k=1}^{n} b_{r}\left(\mathbf{x}_{k}^{\perp}\right),
$$

where $\psi$ is the ground state of a one-dimensional Bose gas of particles with hard cores of radius $R_{0}$. It is well known that it's energy is the same as that of $n$ non-interacting fermions on a line of reduced length $\ell-(n-1) R_{0}$. An explicit calculation yields $(3.5)$.

\subsection{Lower bound for Theorem 3.2}

We start by defining an auxiliary 2D GP energy functional by

$$
\phi \mapsto \int_{\mathbb{R}^{2}}\left(\left|\nabla^{\perp} \phi\right|^{2}+V^{\perp}|\phi|^{2}+p|\phi|^{4}\right) d^{2} \mathbf{x}^{\perp}
$$

for some parameter $p \geq 0$. The following fact will be needed below.

Lemma 1. For any $p \geq 0$, there exists a unique minimizer (up to a constant phase factor) of (3.70) under the normalization condition $\int|\phi|^{2}=1$, denoted by $\phi_{p}$, that can be chosen strictly positive. Moreover, both $\phi_{p}$ and $\nabla^{\perp} \phi_{p}$ are bounded uniformly in $p$ and $\mathbf{x}^{\perp}$ for $p$ in a finite interval $[0, P]$.

The proof of this Lemma, as well as the proof of Lemmas 2 and 3 below, will be given in the Appendix.

The energy corresponding to the minimizer $\phi_{p}$ will be denoted by $E^{\text {aux }}(p)$. Define also $\phi_{p, r}\left(\mathbf{x}^{\perp}\right)=r^{-1} \phi_{p}\left(\mathbf{x}^{\perp} / r\right)$. Writing the wave function as

$$
\Psi\left(\mathbf{x}_{1}, \ldots, \mathbf{x}_{n}\right)=f\left(\mathbf{x}_{1}, \ldots, \mathbf{x}_{n}\right) \prod_{k=1}^{n} \phi_{p, r}\left(\mathbf{x}_{k}^{\perp}\right),
$$

using partial integration and the variational equation for $\phi_{p}$, we obtain

$$
\begin{aligned}
& \langle\Psi|H| \Psi\rangle-\frac{n}{r^{2}} E^{\text {aux }}(p)-\frac{n p}{r^{2}} \int \phi_{p}^{4} \\
& =\sum_{i=1}^{n} \int\left[\left|\nabla_{i} f\right|^{2}+\frac{1}{2} \sum_{j, j \neq i} v_{a}\left(\left|\mathbf{x}_{i}-\mathbf{x}_{j}\right|\right)|f|^{2}-2 p \phi_{p, r}\left(\mathbf{x}_{i}^{\perp}\right)^{2}|f|^{2}\right] \prod_{k=1}^{n} \phi_{p, r}\left(\mathbf{x}_{k}^{\perp}\right)^{2} d^{3} \mathbf{x}_{k} .
\end{aligned}
$$

We now divide space in $\mathbf{x}^{\perp}$-direction into boxes of side length $s$, labeled by $\alpha$. Let $\phi_{\alpha, \max }$ and $\phi_{\alpha, \min }$ denote the maximal and minimal value of $\phi_{p, r}$ inside box $\alpha$, respectively. We obtain

$$
E_{\mathrm{N}}^{\mathrm{QM}}(n, \ell, r, a)-\frac{n}{r^{2}} E^{\mathrm{aux}}(p)-\frac{n p}{r^{2}} \int \phi_{p}^{4} \geq \inf _{\left\{n_{\alpha}\right\}} \sum_{\alpha}\left[E_{\alpha}\left(n_{\alpha}\right)-2 n_{\alpha} p \phi_{\alpha, \max }^{2}\right]
$$


with

$$
E_{\alpha}(n)=\inf _{f} \sum_{i=1}^{n} \frac{\int_{\alpha}\left[\left|\nabla_{i} f\right|^{2}+\frac{1}{2} \sum_{j, j \neq i} v_{a}\left(\left|\mathbf{x}_{i}-\mathbf{x}_{j}\right|\right)|f|^{2}\right] \prod_{k=1}^{n} \phi_{p, r}\left(\mathbf{x}_{k}^{\perp}\right)^{2} d^{3} \mathbf{x}_{k}}{\int_{\alpha}|f|^{2} \prod_{k=1}^{n} \phi_{p, r}\left(\mathbf{x}_{k}^{\perp}\right)^{2} d^{3} \mathbf{x}_{k}} .
$$

In (3.73) the infimum is over all possible distributions of the $n$ particles into the boxes $\alpha$. As a first step we will replace $\phi_{\alpha, \max }^{2}$ by $\phi_{\alpha, \min }^{2}$ in the last term in (3.73). The error in doing so is bounded above by

$$
2 n p \sup _{\alpha}\left(\phi_{\alpha, \max }^{2}-\phi_{\alpha, \min }^{2}\right) \leq 2 \sqrt{2} n \frac{p s}{r^{3}}\left\|\nabla^{\perp} \phi_{p}^{2}\right\|_{\infty} .
$$

To bound $E_{\alpha}$ from below, we divide $\alpha$ into even smaller boxes, denoted by $\beta$, with side length $t \leq s$, where $s / t \in \mathbb{N}$. Let $c_{i}$ denote the number of boxes with exactly $i$ particles. Then

$$
E_{\alpha}(n) \geq \inf _{\left\{c_{i}\right\}} \sum_{i \geq 0} c_{i} \inf _{\beta \subset \alpha} E_{\beta}(i)
$$

Note that the infimum is under the constraints $\sum c_{i}=(s / t)^{2}$ and $\sum c_{i} i=n$. For a lower bound on $E_{\beta}$, we use

$$
E_{\beta}(n) \geq\left(\frac{\phi_{\beta, \text { min }}^{2}}{\phi_{\beta, \max }^{2}}\right)^{n} \inf _{f} \sum_{i=1}^{n} \frac{\int_{\beta}\left[\left|\nabla_{i} f\right|^{2}+\frac{1}{2} \sum_{j, j \neq i} v_{a}\left(\left|\mathbf{x}_{i}-\mathbf{x}_{j}\right|\right)|f|^{2}\right] \prod_{k=1}^{n} d^{3} \mathbf{x}_{k}}{\int_{\beta}|f|^{2} \prod_{k=1}^{n} d^{3} \mathbf{x}_{k}} .
$$

Fix some $\delta>0$, and assume that $\phi_{\alpha, \min }^{2} \geq \delta / r^{2}$. Then

$$
\left(\frac{\phi_{\beta, \min }^{2}}{\phi_{\beta, \max }^{2}}\right)^{n} \geq 1-\sqrt{2} n \frac{t}{r} \frac{\left\|\nabla^{\perp} \phi_{p}^{2}\right\|_{\infty}}{\delta}
$$

(compare with (3.35)). The rest of (3.77) can be bounded below by the same method as in the previous subsection. The only difference lies in the fact that $b_{r}$ is replaced by the constant function, and the integrations are only over the box $\beta$. The result is (compare with (3.2))

$$
\begin{aligned}
E_{\beta}(n) \geq & E_{\mathrm{N}}^{1 \mathrm{D}}\left(n, \ell, 8 \pi a / t^{2}\right) \\
& \cdot\left(1-C n\left(\frac{a}{t}\right)^{1 / 8}\left[1+\frac{n t}{\ell}\left(\frac{a}{t}\right)^{1 / 8}\right]\right)\left(1-\sqrt{2} n \frac{t}{r} \frac{\left\|\nabla \phi_{p}^{2}\right\|_{\infty}}{\delta}\right) .
\end{aligned}
$$

To proceed we need an explicit lower bound on $E_{\mathrm{N}}^{1 \mathrm{D}}$ that will be proved in the Appendix.

Lemma 2. There is a finite number $C>0$ such that

$$
E_{\mathrm{N}}^{1 \mathrm{D}}(n, \ell, g) \geq \frac{1}{2} \frac{n(n-1)}{\ell} g\left(1-C n(\ell g)^{1 / 2}\right) .
$$


Applying this lemma to (3.79), we obtain

$$
\begin{aligned}
E_{\beta}(n) \geq & \frac{n(n-1)}{\ell} \frac{4 \pi a}{t^{2}}\left(1-C n\left(\frac{a}{t}\right)^{1 / 8}\left[1+\frac{n t}{\ell}\left(\frac{a}{t}\right)^{1 / 8}\right]\right) \\
& \cdot\left(1-\sqrt{2} n \frac{t}{r} \frac{\left\|\nabla^{\perp} \phi_{p}^{2}\right\|_{\infty}}{\delta}\right)\left(1-C n\left(\ell a / t^{2}\right)^{1 / 2}\right) .
\end{aligned}
$$

Note that the right side is independent of $\beta \subset \alpha$. We insert this bound in (3.76), and use the following Lemma. It is a simple generalization of a result of [31]. Although we need at this point only the version proved in [31], we state the Lemma in this general form for later use.

Lemma 3 ([31]). For $n \in \mathbb{N} \cup\{0\}$, let $E(n)$ be a sequence of non-negative real numbers that is superadditive, i.e., $E\left(n_{1}+n_{2}\right) \geq E\left(n_{1}\right)+E\left(n_{2}\right)$, and bounded below by

$$
E(n) \geq L(n) K(n),
$$

with $K: \mathbb{R}_{+} \rightarrow \mathbb{R}_{+}$monotone decreasing, $L: \mathbb{R}_{+} \rightarrow \mathbb{R}_{+}$convex, $L(0)=0$, and

$$
L^{\prime}(x) \leq \frac{L(\lambda x)}{2 \lambda x}
$$

for some $\lambda>1$ and all $x>0$. (Here $L^{\prime}$ denotes the right derivative of L.) Let $c_{n}$ be a sequence of non-negative real numbers, with

$$
\sum_{n \geq 0} c_{n} \leq M \quad \text { and } \sum_{n \geq 0} c_{n} n=N
$$

Then

$$
\sum_{n \geq 0} c_{n} E(n) \geq M L(N / M) K(\langle\lambda N / M\rangle)
$$

where $\langle x\rangle$ denotes the smallest integer $\geq x$.

The proof is given in the Appendix. Note that $\inf _{\beta} E_{\beta}(n)$ is certainly a superadditive function, as the infimum over superadditive functions. Therefore we can apply Lemma 3 with $L(x)=x[x-1]_{+}$and $\lambda=4$, to (3.76), together with (3.81), and obtain

$$
E_{\alpha}\left(n_{\alpha}\right) \geq \frac{4 \pi a n_{\alpha}^{2}}{\ell s^{2}}\left(1-\frac{1}{n_{\alpha}} \frac{s^{2}}{t^{2}}\right) \mathcal{R}\left(n_{\alpha}\right)
$$

where

$$
\begin{aligned}
\mathcal{R}(n)= & \left(1-C\left\langle 4 n t^{2} / s^{2}\right\rangle\left(\frac{a}{t}\right)^{1 / 8}\left(1+\left\langle 4 n t^{2} / s^{2}\right\rangle \frac{t}{\ell}\left(\frac{a}{t}\right)^{1 / 8}\right)\right) \\
& \cdot\left(1-\sqrt{2}\left\langle 4 n t^{2} / s^{2}\right\rangle \frac{t}{r} \frac{\left\|\nabla^{\perp} \phi_{p}^{2}\right\|_{\infty}}{\delta}\right)\left(1-C\left\langle 4 n t^{2} / s^{2}\right\rangle\left(\ell a / t^{2}\right)^{1 / 2}\right) .
\end{aligned}
$$


We now insert this bound in (3.73), and use $n_{\alpha} \leq n$ in the error terms. Note that $\mathcal{R}$ is monotone decreasing in $n$, hence $\mathcal{R}\left(n_{\alpha}\right) \geq \mathcal{R}(n)$. Minimizing over $n_{\alpha}$ gives

$$
E_{\alpha}\left(n_{\alpha}\right)-2 n_{\alpha} p \phi_{\alpha, \min }^{2} \geq-\frac{\ell s^{2} p^{2} \phi_{\alpha, \min }^{4}}{4 \pi a}\left(1+\frac{2 \pi a r^{2}}{\ell t^{2} p \delta}\right)^{2} \frac{1}{\mathcal{R}(n)} .
$$

This holds for boxes $\alpha$ where $\phi_{\alpha, \min }^{2} \geq \delta / r^{2}$. In boxes where this is not the case, we simply use positivity of $E_{\alpha}$ (which follows from positivity of $v_{a}$ ) in the form

$$
E_{\alpha}\left(n_{\alpha}\right)-2 n_{\alpha} p \phi_{\alpha, \min }^{2} \geq-2 n_{\alpha} \frac{p \delta}{r^{2}} .
$$

Putting everything together, using $\sum_{\alpha} s^{2} \phi_{\alpha, \text { min }}^{4} \leq r^{-2} \int \phi_{p}^{4}$ and choosing

$$
p=\frac{4 \pi a n}{\ell},
$$

we obtain

$$
\begin{aligned}
& E_{\mathrm{N}}^{\mathrm{QM}}(n, \ell, r, a) \geq \frac{n}{r^{2}} E^{\mathrm{aux}}(4 \pi a n / \ell) \\
& -\frac{4 \pi a n^{2}}{\ell r^{2}}\left(\sqrt{8}\left\|\nabla^{\perp} \phi_{p}^{2}\right\|_{\infty} \frac{s}{r}+2 \delta+\int \phi_{p}^{4}\left[\left(1+\frac{r^{2}}{2 t^{2} \delta n}\right)^{2} \frac{1}{\mathcal{R}(n)}-1\right]\right) .
\end{aligned}
$$

We are still free to choose the parameters $t, s$ and $\delta$.

It remains to derive a lower bound on $E^{\text {aux }}$. This will be done similarly to the lower bound on the 3D GP energy given in Subsect. 2.7. Consider the auxiliary Schrödinger operator

$$
H^{\text {aux }}=-\Delta^{\perp}+V^{\perp}\left(\mathbf{x}^{\perp}\right)+2 p b\left(\mathbf{x}^{\perp}\right)^{2} .
$$

Using $\phi_{p}$ as a trial function, we have

$$
\begin{aligned}
\inf \operatorname{spec} H^{\text {aux }} & \leq E^{\text {aux }}(p)-p \int \phi_{p}^{4}+2 p \int b^{2} \phi_{p}^{2} \\
& \leq E^{\operatorname{aux}}(p)+p \int b^{4} .
\end{aligned}
$$

On the other hand, using Temple's inequality (2.61),

$$
\begin{aligned}
\inf \operatorname{spec} H^{\text {aux }} & \geq e^{\perp}+2 p \int b^{4}-\frac{4 p^{2} \int b^{6}}{\widetilde{e}^{\perp}-2 p \int b^{4}} \\
& \geq e^{\perp}+2 p \int b^{4}\left(1-\frac{2 p\|b\|_{\infty}^{2}}{\widetilde{e}^{\perp}-2 p\|b\|_{\infty}^{2}}\right) .
\end{aligned}
$$

Eqs. (3.93) and (3.94) together give

$$
E^{\operatorname{aux}}(p) \geq e^{\perp}+p \int b^{4}\left(1-\frac{4 p\|b\|_{\infty}^{2}}{\widetilde{e}^{\perp}-2 p\|b\|_{\infty}^{2}}\right) .
$$


We now choose

$$
s=\epsilon r, \quad t=\frac{r}{\epsilon \sqrt{n}}, \quad \delta=\epsilon
$$

and

$$
\epsilon=n^{-1 / 14}+\left(\frac{n \ell a}{r^{2}}\right)^{1 / 8}+\left(\left[1+\frac{r}{\sqrt{n} \ell}\right]\left(\frac{\sqrt{n} a}{r}\right)^{1 / 4}\right)^{4 / 39}
$$

and obtain as the final result

$$
E_{\mathrm{N}}^{\mathrm{QM}}(n, \ell, r, a) \geq \frac{n e^{\perp}}{r^{2}}+\frac{n^{2} g}{2 \ell}\left(1-C\left[\epsilon+\frac{n a}{\ell}\right]\right) .
$$

Note that we did not pay any attention to the restriction $s / t \in \mathbb{N}$ in choosing $s$ and $t$. However, since, with our choice, $s / t=\epsilon^{2} \sqrt{n} \geq n^{1 / 2-1 / 7}$, this can easily be made an integer by replacing $\epsilon$ by some $\bar{\epsilon}$ with $\epsilon \leq \bar{\epsilon} \leq 2 \epsilon$. This affects only the constant $C$ in (3.98).

\subsection{Boundary conditions}

As a last step in this section, before giving the proof of our main Theorem 1.1, we investigate the dependence of the ground state energy of (1.3) on the boundary conditions. In the upper bound above we used Dirichlet boundary conditions for the energy $E^{1 \mathrm{D}}$, and Neumann boundary conditions for the lower bound. To relate these energies to the energy with periodic boundary conditions and to prove independence of boundary conditions in the thermodynamic limit, we need the following Lemma.

Lemma 4. Denote $E_{\mathrm{p}}^{1 \mathrm{D}}(n, \ell, g)$ the ground state energy of (1.3) with periodic boundary conditions, i.e., on the torus $[0, \ell]^{n}$. Then there is a finite number $C>0$ such that

$$
E_{\mathrm{N}}^{1 \mathrm{D}}(n, \ell, g) \leq E_{\mathrm{p}}^{1 \mathrm{D}}(n, \ell, g) \leq E_{\mathrm{D}}^{1 \mathrm{D}}(n, \ell, g)
$$

and

$$
E_{\mathrm{D}}^{1 \mathrm{D}}(n, \ell, g) \leq E_{\mathrm{N}}^{1 \mathrm{D}}(n, \ell, g)+C \frac{n^{7 / 3}}{\ell^{2}}
$$

Proof. The first inequality (3.99) is standard, noting that the interaction considered has zero range. For (3.100) we use a result of [37, Lemma 2.1.13 and Prop. 2.2.10], which implies, for $0<b<\ell / 2$,

$$
E_{\mathrm{D}}^{1 \mathrm{D}}(n, \ell+2 b, g) \leq E_{\mathrm{N}}^{1 \mathrm{D}}(n, \ell, g)+\frac{2 n}{b^{2}} .
$$

Using $E_{\mathrm{D}}^{1 \mathrm{D}}(n, \ell+2 b, g)=E_{\mathrm{D}}^{1 \mathrm{D}}(n, \ell, g(1+2 b / \ell))(1+2 b / \ell)^{-2} \geq E_{\mathrm{D}}^{1 \mathrm{D}}(n, \ell, g)(1+2 b / \ell)^{-2}$ and $E_{\mathrm{N}}^{1 \mathrm{D}}(n, \ell, g) \leq \pi^{2} n^{3} /\left(3 \ell^{2}\right)$, we obtain

$$
E_{\mathrm{D}}^{1 \mathrm{D}}(n, \ell, g) \leq E_{\mathrm{N}}^{1 \mathrm{D}}(n, \ell, g)+\frac{2 n}{b^{2}}(1+2 b / \ell)^{2}+\frac{n^{3}}{\ell^{2}} \frac{4 \pi^{2}}{3} \frac{b}{\ell}(1+b / \ell) .
$$

Now $b=$ const. $\ell / n^{2 / 3}$ gives the desired result. 


\section{Proof of Theorem 1.1}

With the results of the previous section in hand, we can now give the proof of our main Theorem 1.1. The proof will be divided into four subsections, two for the upper and lower bound, respectively. In each subsection, we compare the ground state energy of $H_{N, L, r, a}$ with the ground state energy of one of the functionals in Subsections 2.12.5, which, as explained there and in Subsection 2.6, is asymptotically equal to $E(N, L, g)$ in the respective parameter region. Combining all the bounds obtained, this will prove Theorem 1.1, together with the claimed uniformity in the parameters. The corresponding convergence of the ground state particle density, as stated in Theorems 2.1-2.5, follows in a standard way by variation with respect to the external potential $V_{L}(z)$ (compare with Props. 2.1 and 2.2 in Subsect. 2.6). Since the proof of the energy convergence is already quite lengthy by itself, the simple modifications necessary for a proof of the density convergence will be omitted.

Let again $\bar{L}=N / \bar{\rho}$ denote the extension of the system in $z$-direction. As already mentioned in the beginning of Section 3, it will be necessary, for the lower bound to $E^{\mathrm{QM}}$, to consider the case of small and large $N r / \bar{L}$ separately. We will divide space in $z$-directions into small boxes, and use the bounds of Section 3 in each box. To control the number of particles in each box, Lemma 3 will be essential. For small $N r / \bar{L}$, where $r$ is smaller than the mean particle distance, we will use the lower bound given in Thm. 3.1. For larger values of $N r / \bar{L}$, where $r$ is actually bigger than the mean particle distance, the lower bound of Thm. 3.2 will be used instead. Note that this distinction is only relevant in Regions $1-3$, since in Regions 4 and $5 \mathrm{Nr} / \bar{L}=r \bar{\rho} \ll 1$ by condition (1.12). Hence $r$ is always smaller than the mean particle distance in Regions 4 and 5 .

\subsection{Upper bound for Regions 1-3}

For an upper bound that gives the right asymptotics as long as $g / \bar{\rho} \ll 1$, we can essentially use the same technique as in [27]. The results of Section 3 are not needed in this case. As a trial function, we use

$$
\Psi\left(\mathbf{x}_{1}, \ldots, \mathbf{x}_{N}\right)=F\left(\mathbf{x}_{1}, \ldots, \mathbf{x}_{N}\right) \prod_{k=1}^{N} b_{r}\left(\mathbf{x}_{k}^{\perp}\right) \phi^{\mathrm{GP}}\left(z_{k}\right),
$$

where $\phi^{\mathrm{GP}}=\left(\rho_{N, L, g}^{\mathrm{GP}}\right)^{1 / 2}$ (cf. Subsect. 2.2), and $F$ is the 'Dyson wave function', described in $[9,27]$. The result is

$$
E^{\mathrm{QM}}(N, L, r, a) \leq \frac{N e^{\perp}}{r^{2}}+E^{\mathrm{GP}}(N, L, g)\left(1+C a\left\|b_{r}\right\|_{\infty}^{2 / 3}\left\|\phi^{\mathrm{GP}}\right\|_{\infty}^{2 / 3}\right)
$$

as long as $a\left\|b_{r}\right\|_{\infty}^{2 / 3}\left\|\phi^{\mathrm{GP}}\right\|_{\infty}^{2 / 3} \leq 1$. Note that, by the same proof as in Lemma 2.1 of $[28], g\left\|\phi^{\mathrm{GP}}\right\|_{\infty}^{2} \leq 2 E^{\mathrm{GP}} / N$, and therefore

$$
E^{\mathrm{QM}}(N, L, r, a) \leq \frac{N e^{\perp}}{r^{2}}+E^{\mathrm{GP}}(N, L, g)\left(1+\text { const. } a^{2 / 3} N^{-1 / 3} E^{\mathrm{GP}}(N, L, g)^{1 / 3}\right) .
$$


Now, in Regions $1-3, E^{\mathrm{GP}}(N, L, g) \sim E(N, L, g)$, and

$$
a^{2} E^{\mathrm{GP}}(N, L, g) / N \sim a^{2}\left(L^{-2}+g \bar{\rho}\right) \sim(a / L)^{2}+\frac{g}{\bar{\rho}}\left(g \bar{\rho} r^{2}\right)^{2} \ll 1 .
$$

\subsection{Upper bound for Regions 4 and 5}

If $g / \bar{\rho}$ is not small, the method of the previous subsection does not work, and we have to proceed differently. As in Subsect. 3.5, let $E_{\mathrm{p}}^{1 \mathrm{D}}(n, \ell, g)$ denote the ground state energy of (1.3) on an interval of length $\ell$, with periodic boundary conditions, and write $E_{\mathrm{p}}^{1 \mathrm{D}}(n, \ell, g)=n^{3} e_{n}(g \ell / n) / \ell^{2}$. In [22] it is shown that, for every fixed $t \geq 0$, $\lim _{n \rightarrow \infty} e_{n}(t)=e(t)$. Since the functions are monotone increasing, concave and bounded in $t$, the convergence is actually uniform in $t$. By Lemma 4 of Subsection 3.5 , the same is true with $E_{\mathrm{D}}^{1 \mathrm{D}}$ instead of $E_{\mathrm{p}}^{1 \mathrm{D}}$. Hence we get the estimate

$$
E_{\mathrm{D}}^{1 \mathrm{D}}(n, \ell, g) \leq \frac{n^{3}}{\ell^{2}}(e(g \ell / n)+\delta(n))
$$

for some bounded function $\delta$ satisfying $\lim _{n \rightarrow \infty} \delta(n)=0$. Without loss of generality we may assume that $\delta$ is monotone decreasing.

Let $\rho$ be the minimizer of the LL functional (2.19) under the normalization condition $\int \rho=N$. Note that $\rho$ has compact support, with radius $R=L\left(\mu L^{2}\right)^{1 / s}$, where $\mu=\partial E^{\mathrm{LL}}(N, L, g) / \partial N$. (This $R$ is different from that in Eq. (3.27).) By monotonicity and concavity of $E^{\mathrm{LL}}$ in $g$, and by the scaling relation (2.22),

$$
\frac{E^{\mathrm{LL}}(N, L, g)}{N} \leq \mu \leq 3 \frac{E^{\mathrm{LL}}(N, L, g)}{N}
$$

Divide $\mathbb{R}$ in $z$-direction into intervals of length $\ell$, labeled by $\alpha$, with $R_{0}<\ell<R / 2$. Let $n_{\alpha} \in \mathbb{N}$ be a collection of integers such that $\sum_{\alpha} n_{\alpha}=N$. Let $V_{\alpha}=\sup _{z \in \alpha} V_{L}(z)$,

and denote $E_{\mathrm{D}}^{\mathrm{QM}}(n, \ell, r, a)$ the ground state energy of (3.1) in a box of side length $\ell$ and with Dirichlet boundary conditions. By confining the particles into different boxes of length $\ell-R_{0}$, a distance $R_{0}$ apart, we get the estimate

$$
E^{\mathrm{QM}}(N, L, r, a) \leq \sum_{\alpha}\left[E_{\mathrm{D}}^{\mathrm{QM}}\left(n_{\alpha}, \ell-R_{0}, r, a\right)+V_{\alpha} n_{\alpha}\right]
$$

Using (3.3) and (4.5) as well as monotonicity of $e$, we obtain

$$
E^{\mathrm{QM}}(N, L, r, a)-\frac{N e^{\perp}}{r^{2}} \leq \sum_{\alpha}\left[\frac{n_{\alpha}^{3}}{\ell^{2}}\left(e\left(g \ell / n_{\alpha}\right)+\delta\left(n_{\alpha}\right)\right) \mathcal{R}\left(n_{\alpha}\right)+V_{\alpha} n_{\alpha}\right]
$$

with

$$
\mathcal{R}(n)=\frac{1}{\left(1-R_{0} / \ell\right)^{2}}\left(1+C\left[\left(\frac{n a}{r}\right)^{2}(1+g \ell)\right]^{1 / 3}\right),
$$

provided we choose $n_{\alpha}$ and $\ell$ such that the term in square brackets is less than 1 . Note the additional factor $\left(1-R_{0} / \ell\right)^{-2}$, which is due to the fact that the size of the 
box is only $\ell-R_{0}$. Now let $\bar{n}_{\alpha}=\int_{\alpha} \rho(z) d z$, and $n_{\alpha}=\left\langle\bar{n}_{\alpha}\right\rangle$, where $\langle x\rangle$ denotes the smallest integer $\geq x$. With this choice $\sum_{\alpha} n_{\alpha} \geq N$, but by monotonicity of (4.8) in $N$ we can plug in these values of $n_{\alpha}$ for an upper bound.

Since $n_{\alpha} \leq \bar{n}_{\alpha}+1$, and $e$ and $\delta$ are monotone increasing and decreasing, respectively, we obtain

$$
(4.8) \leq \sum_{\alpha}\left[\frac{\bar{n}_{\alpha}^{3}}{\ell^{2}}\left(e\left(g \ell / \bar{n}_{\alpha}\right)+\delta\left(\bar{n}_{\alpha}\right)\right)\left(1+\frac{1}{\bar{n}_{\alpha}}\right)^{3} \mathcal{R}\left(\ell\|\rho\|_{\infty}+1\right)+V_{\alpha}\left(\bar{n}_{\alpha}+1\right)\right] .
$$

Here we estimated $\bar{n}_{\alpha}$ by $\ell\|\rho\|_{\infty}$ in $\mathcal{R}$. Denote $\hat{V}_{\alpha}=\min _{z \in \alpha} V_{L}(z)$. We estimate $V_{\alpha} \leq \hat{V}_{\alpha}+$ const. $L^{-2}(\ell / L)(R / L)^{s-1}$ in boxes where $n_{\alpha}>0$. Using $(R / L)^{s} \leq$ $3 L^{2} E^{\mathrm{LL}}(N, L, g) / N$ (see (4.6)), we therefore see that the error in replacing $V_{\alpha}$ by $\hat{V}_{\alpha}$ is, in total, bounded above by const. $E^{\mathrm{LL}}(N, L, g)(\ell / R)$.

Fix some $0<\epsilon<1$. We first bound the contribution to (4.10) from boxes where $\bar{n}_{\alpha} \leq 1 / \epsilon$. Now both $e$ and $\delta$ are bounded, the number of boxes with nonzero $\bar{n}_{\alpha}$ is bounded by $(R / \ell)+2$, and $\hat{V}_{\alpha} \leq L^{-2}(R / L)^{s}$ in these boxes. Therefore this contribution is bounded above by

$$
\text { const. } \frac{R}{\epsilon^{2} \ell}\left(\frac{1}{\epsilon \ell^{2}} \mathcal{R}\left(\ell\|\rho\|_{\infty}+1\right)+\epsilon \frac{E^{\mathrm{LL}}(N, L, g)}{N}\right) \text {. }
$$

For the remaining boxes, we use $\bar{n}_{\alpha} \geq 1 / \epsilon$ and $\bar{n}_{\alpha} \leq \ell\|\rho\|_{\infty}$ to obtain

$$
\begin{aligned}
(4.10) \leq & (4.11)+\text { const. } E^{\mathrm{LL}}(N, L, g) \frac{\ell}{R} \\
& +(1+\epsilon) \cdot \sum_{\alpha}\left[\frac{n_{\alpha}^{3}}{\ell^{2}}\left(e\left(g \ell / n_{\alpha}\right)+\delta(1 / \epsilon)\right)(1+\epsilon)^{2} \mathcal{R}\left(\ell\|\rho\|_{\infty}+1\right)+\hat{V}_{\alpha} n_{\alpha}\right] .
\end{aligned}
$$

Since $x \mapsto x^{3} e(1 / x)$ is convex, we can use Jensen's inequality to bound the sum from above by

$$
\int_{\mathbb{R}}\left[\rho(z)^{3}(e(g / \rho(z))+\delta(1 / \epsilon))(1+\epsilon)^{2} \mathcal{R}\left(\ell\|\rho\|_{\infty}+1\right)+V(z) \rho(z)\right] d z .
$$

Now, by the scaling (2.23), $\rho(z)=\gamma \tilde{\rho}_{g / \gamma}(\gamma z / N)$, where $\gamma=(N / L) N^{-2 /(s+2)}$ and $\tilde{\rho}_{g / \gamma}$ is a function that depends only on $g / \gamma$. Therefore $\|\rho\|_{\infty} \leq \gamma\left\|\tilde{\rho}_{g / \gamma}\right\|_{\infty}$, and $\|\rho\|_{3}^{3} \leq N \gamma^{2}\left\|\tilde{\rho}_{g / \gamma}\right\|_{\infty}^{2}$

We choose, for some $0<\hat{\epsilon}<\epsilon, \ell=(\hat{\epsilon} \gamma)^{-1}$, and use

$$
E^{\mathrm{LL}}(1,1, g / \gamma)^{1 / s} \leq \frac{\gamma R}{N} \leq \pi^{2 / s}
$$

Putting everything together, we get, for $C$ denoting some universal constant,

$$
\begin{aligned}
& E^{\mathrm{QM}}(N, L, r, a)-\frac{N e^{\perp}}{r^{2}} \\
& \leq\left(E^{\mathrm{LL}}(N, L, g)+N \gamma^{2}\left(\left\|\tilde{\rho}_{g / \gamma}\right\|_{\infty}^{2} \delta(1 / \epsilon)+C(\hat{\epsilon} / \epsilon)^{3}\right)\right) \cdot \mathcal{R}^{\prime},
\end{aligned}
$$


where

$$
\begin{aligned}
\mathcal{R}^{\prime}= & \left(1+\frac{C}{N \hat{\epsilon} E^{\mathrm{LL}}(1,1, g / \gamma)^{1 / s}}+C \frac{\hat{\epsilon}}{\epsilon}\right)(1+\epsilon)^{3} \\
& \cdot\left(1-\frac{a R_{0}}{r^{2}} \hat{\epsilon} \frac{\gamma}{g}\right)^{-2}\left(1+C\left(\frac{a\left\|\tilde{\rho}_{g / \gamma}\right\|_{\infty}}{\hat{\epsilon} r}\right)^{2 / 3}\left(1+\frac{g}{\hat{\epsilon} \gamma}\right)^{1 / 3}\right)
\end{aligned}
$$

The choice of $\epsilon$ and $\hat{\epsilon}$ is determined by $a / r, g / \gamma$ and $N$. The bound is uniform in $g / \gamma$ for bounded $g / \gamma$ and $\gamma / g$.

If $g / \gamma \rightarrow \infty$ as $N \rightarrow \infty$ (Region 5), we can use the same method to obtain an upper bound, replacing the bound (3.3) by (3.5) in (4.8). This gives a bound uniform in $g / \gamma$ (for $\gamma / g$ bounded). Combined with the result (4.15), this shows that in the limit $N \rightarrow \infty$ and $r / L \rightarrow 0$

$$
\limsup \frac{E^{\mathrm{QM}}(N, L, r, a)-N e^{\perp} / r^{2}}{E^{\mathrm{LL}}(N, L, g)} \leq 1,
$$

uniformly in the parameters, as long as $a / r \rightarrow 0$ and $\gamma / g$ stays bounded.

\subsection{Lower bound for Regions 3-5}

We now derive a lower bound on $E^{\mathrm{QM}}$ that will give the right asymptotics in Regions 3-5. As in the upper bound, given in Subsection 4.2, we will use the box method, this time with Neumann boundary conditions. In each box, the results of Section 3 will be used. In analogy to (4.5) we infer from [22] and Lemma 4 that

$$
E_{\mathrm{N}}^{1 \mathrm{D}}(n, \ell, g) \geq \frac{n^{3}}{\ell^{2}}(e(g \ell / n)-\delta(n))
$$

for some bounded, monotone decreasing function $\delta$ satisfying $\lim _{n \rightarrow \infty} \delta(n)=0$. This will be used in the bound for Regions 4 and 5 . If $g \ell / n$ is small, however, we use $e(g \ell / n) \leq \frac{1}{2} g \ell / n$ and $(3.80)$ to obtain

$$
E_{\mathrm{N}}^{1 \mathrm{D}}(n, \ell, g) \geq \frac{n^{2}(n-1)}{\ell^{2}} e(g \ell / n)\left(1-C n(g \ell)^{1 / 2}\right) .
$$

We divide $\mathbb{R}$ in $z$-direction into intervals of side length $M$, labeled by $\alpha$. Denote $E_{\mathrm{N}}^{\mathrm{QM}}(n, M, r, a)$ the ground state energy of (3.1) in a box of side length $M$ and with Neumann boundary conditions. Let again $\hat{V}_{\alpha}=\inf _{z \in \alpha} V_{L}(z)$, and $V_{\alpha}=\sup _{z \in \alpha} V_{L}(z)$. By confining the particles into different boxes and neglecting the interaction between different boxes, we get the estimate

$$
E^{\mathrm{QM}}(N, L, r, a) \geq \inf _{\left\{N_{\alpha}\right\}} \sum_{\alpha}\left[E_{\mathrm{N}}^{\mathrm{QM}}\left(N_{\alpha}, M, r, a\right)+\hat{V}_{\alpha} N_{\alpha}\right]
$$

where the infimum is over all distributions of the $N$ particles among the boxes $\alpha$. As in the upper bound, we can estimate the difference of the maximum and minimum of $V_{L}$ for boxes alpha $\alpha$ inside some interval $[-R, R]$ by const. $L^{-2}(M / L)(R / L)^{s-1}$. 
For boxes outside $[-R, R]$ we use $\hat{V}_{\alpha} \geq V_{\alpha}(1-s M / R)$. Choosing $R$ the radius of the LL minimizer, and $M=\epsilon R$ for some $0<\epsilon<1$, we see that, analogously to the upper bound, the error in replacing $\hat{V}_{\alpha}$ by $V_{\alpha}(1-s \epsilon)$ is, in total, bounded above by const. $\epsilon E^{\mathrm{LL}}(N, L, g)$.

We now have to estimate $E_{\mathrm{N}}^{\mathrm{QM}}\left(N_{\alpha}, M, r, a\right)$ from below. We cannot directly use (3.2), because this bound is not uniform in the particle number. Instead we proceed similarly to [31]. We divide the interval $M$ again into smaller intervals of length $\ell=\eta M$, where $1 / \eta \in \mathbb{N}$. Neglecting the interaction between different boxes, we obtain

$$
E_{\mathrm{N}}^{\mathrm{QM}}\left(N_{\alpha}, M, r, a\right) \geq \inf _{\left\{c_{n}\right\}} \sum_{n=1}^{N_{\alpha}} c_{n} E_{\mathrm{N}}^{\mathrm{QM}}(n, \ell, r, a),
$$

where $c_{n}$ denotes the number of boxes containing exactly $n$ particles, and the infimum is over all $c_{n} \in \mathbb{N}$ under the condition $\sum_{n} c_{n} n=N_{\alpha}$ and $\sum_{n} c_{n}=M / \ell=\eta^{-1}$.

Fix some $0<\chi<1$, and consider the case $n \geq 1 / \chi$. Then, using (3.2) and (4.18),

$$
E_{\mathrm{N}}^{\mathrm{QM}}(n, \ell, r, a)-\frac{n e^{\perp}}{r^{2}} \geq \frac{n^{3}}{\ell^{2}} e(g \ell / n)(1-\nu(n)),
$$

with

$$
\nu(n)=\frac{\delta(1 / \chi)}{e(g \ell / n)}+C n\left(\frac{a}{r}\right)^{1 / 8}\left[1+\frac{n r}{\ell}\left(\frac{a}{r}\right)^{1 / 8}\right] .
$$

Note that $\nu(n)$ is monotone increasing in $n$. We now use Lemma 3 from Subsection 3.4 , with $L(x)=x^{3} e(g \ell / x)$. Note that for this $L$ (3.83) holds with $\lambda=6$, since $e$ is a monotone increasing and concave function, with $e(0)=0$. Let $N^{\prime}=\sum_{n \geq 1 / \chi} c_{n} n$. The contribution from $n \geq 1 / \chi$ to the sum in (4.21) will be bounded below using Lemma 3 and (4.22). For $n<1 / \chi$, we simply use $E_{\mathrm{N}}^{\mathrm{QM}}(n, \ell, r, a) \geq n e^{\perp} / r^{2}$. We thus obtain

$$
E_{\mathrm{N}}^{\mathrm{QM}}\left(N_{\alpha}, M, r, a\right)-\frac{N_{\alpha} e^{\perp}}{r^{2}} \geq \frac{N^{\prime 3}}{M^{2}} e\left(g M / N^{\prime}\right)\left(1-\nu\left(\left\langle 6 N^{\prime} \eta\right\rangle\right)\right) .
$$

Using $N_{\alpha} \geq N^{\prime} \geq N_{\alpha}-1 /(\eta \chi)$, this gives

$$
E_{\mathrm{N}}^{\mathrm{QM}}\left(N_{\alpha}, M, r, a\right)-\frac{N_{\alpha} e^{\perp}}{r^{2}} \geq \frac{N_{\alpha}^{3}}{M^{2}} e\left(g M / N_{\alpha}\right)\left(1-\frac{1}{N_{\alpha} \eta \chi}\right)^{3}\left(1-\nu\left(\left\langle 6 N_{\alpha} \eta\right\rangle\right)\right) .
$$

Now if $\hat{\epsilon} N_{\alpha} \geq 2$ for some $0<\hat{\epsilon}<\chi$, we can choose $\frac{1}{2} \hat{\epsilon} \leq \delta \leq \hat{\epsilon}$ such that $\delta N_{\alpha} \in \mathbb{N}$, and take $\eta=\left(\delta N_{\alpha}\right)^{-1}$. We also use $\langle 6 / \delta\rangle \leq\langle 12 / \hat{\epsilon}\rangle \leq 13 / \hat{\epsilon}$ (since $\left.\hat{\epsilon}<1\right)$. Note that, using (4.14) and $\delta \leq \hat{\epsilon}$,

$$
\frac{\hat{\epsilon}}{13} g \ell \geq \frac{\epsilon}{13} \frac{g}{\gamma} E^{\mathrm{LL}}(1,1, g / \gamma)^{1 / s} \equiv \epsilon \xi(g / \gamma)
$$

Therefore

$$
E_{\mathrm{N}}^{\mathrm{QM}}\left(N_{\alpha}, M, r, a\right)-\frac{N_{\alpha} e^{\perp}}{r^{2}} \geq \frac{N_{\alpha}^{3}}{M^{2}} e\left(g M / N_{\alpha}\right) \mathcal{R},
$$


with

$$
\mathcal{R}=\left(1-\frac{\hat{\epsilon}}{\chi}\right)^{3}\left(1-\frac{\delta(1 / \chi)}{e(\epsilon \xi(g / \gamma))}-\frac{C}{\hat{\epsilon}}\left(\frac{a}{r}\right)^{1 / 8}\left[1+\frac{1}{\epsilon \xi(g / \gamma)} \frac{a}{r}\left(\frac{a}{r}\right)^{1 / 8}\right]\right)
$$

Here we used (4.14) and $N_{\alpha} \leq N$ in the last error term.

The bound (4.27) holds for $\hat{\epsilon} N_{\alpha} \geq 2$. If $N_{\alpha}<2 / \hat{\epsilon}$, however, we use

$$
\frac{N_{\alpha}^{3}}{M^{2}} e\left(g M / N_{\alpha}\right) \leq N_{\alpha} \frac{4 \pi^{2}}{3 \hat{\epsilon}^{2} M^{2}} .
$$

Using these bounds in (4.20), we obtain

$$
\begin{aligned}
& E^{\mathrm{QM}}(N, L, r, a)-\frac{N e^{\perp}}{r^{2}}+C \epsilon E^{\mathrm{LL}}(N, L, g)+N \frac{4 \pi^{2}}{3 \hat{\epsilon}^{2} M^{2}} \\
& \geq \inf _{\left\{N_{\alpha}\right\}} \sum_{\alpha}\left[\frac{N_{\alpha}^{3}}{M^{2}} e\left(g M / N_{\alpha}\right)+V_{\alpha} N_{\alpha}\right] \mathcal{R}(1-s \epsilon) .
\end{aligned}
$$

Note that, by (4.14) and (2.22),

$$
\frac{N}{M^{2}}=\frac{N}{\epsilon^{2} R^{2}} \leq E^{\mathrm{LL}}(N, L, g) \frac{1}{\epsilon^{2} N^{2} E^{\mathrm{LL}}(1,1, g / \gamma)^{1+2 / s}} .
$$

Define $\rho(z)=\sum_{\alpha} N_{\alpha} \chi_{\alpha}(z)$, where $\chi_{\alpha}$ is the characteristic function of the interval $\alpha$. The sum in (4.30) is bounded below by $\mathcal{E}^{\mathrm{LL}}[\rho] \geq E^{\mathrm{LL}}(N, L, g)$, and therefore

$$
E^{\mathrm{QM}}(N, L, r, a)-\frac{N e^{\perp}}{r^{2}} \geq E^{\mathrm{LL}}(N, L, g) \mathcal{R}\left(1-C \epsilon-\frac{4 \pi^{2}}{3 \epsilon^{2} \hat{\epsilon}^{2} N^{2} E^{\mathrm{LL}}(1,1, g / \gamma)^{1+2 / s}}\right) .
$$

The choice of $\epsilon, \hat{\epsilon}$ and $\chi$ is determined by $g / \gamma$ and $a / r$. They can be chosen such that (4.32) gives the correct lower bound in the limit considered, uniformly in $g / \gamma$ for bounded $\gamma / g$. This finishes the proof of the lower bound for Regions 4 and 5 .

If $g / \gamma \rightarrow 0$ as $N \rightarrow \infty$, we can use exactly the same strategy, with (4.19) replacing the bound (4.18). Considering the case $n \geq 1 / \chi,(4.22)$ is still valid, but with $\nu(n)$ replaced by

$$
\nu^{\prime}(n)=\chi+\text { const. } n \sqrt{g \ell}+\text { const. } n\left(\frac{a}{r}\right)^{1 / 8}\left[1+\frac{n r}{\ell}\left(\frac{a}{r}\right)^{1 / 8}\right] .
$$

For $N_{\alpha} \geq \max \left\{2 / \hat{\epsilon}, \epsilon^{2} N\right\}$ we proceed exactly as above. (We recall that $0<\epsilon<1$, and $M=\epsilon R$.) The reason why we have to ensure that $N_{\alpha} \geq \epsilon^{2} N$ is the second term in (4.33), where we want $\ell$ to be small. (Note that we choose $\ell=M /\left(\delta N_{\alpha}\right)$.) For $N_{\alpha}<\max \left\{2 / \hat{\epsilon}, \epsilon^{2} N\right\}$, we replace the bound (4.29) by

$$
\begin{aligned}
\frac{N_{\alpha}^{3}}{M^{2}} e\left(g M / N_{\alpha}\right) & \leq \frac{N_{\alpha}}{\epsilon R} \frac{g}{2} \max \left\{2 / \hat{\epsilon}, \epsilon^{2} N\right\} \\
& \leq \frac{\epsilon}{2} \frac{N_{\alpha}}{N} E^{\mathrm{LL}}(N, L, g)\left(1+\frac{2}{N \epsilon^{2} \hat{\epsilon}}\right) \frac{g / \gamma}{E^{\mathrm{LL}}(1,1, g / \gamma)^{1+1 / s}}
\end{aligned}
$$


where we used $e(x) \leq \frac{1}{2} x$ and (4.14). Note that, for small $g / \gamma$, the last fraction is order 1 . We obtain

$$
E^{\mathrm{QM}}(N, L, r, a)-\frac{N e^{\perp}}{r^{2}} \geq E^{\mathrm{LL}}(N, L, g) \mathcal{R}^{\prime}
$$

with $\mathcal{R}^{\prime}$ given by

$$
\begin{aligned}
\mathcal{R}^{\prime}= & \left(1-\frac{\hat{\epsilon}}{\chi}\right)^{3}\left(1-\chi-C \frac{1}{\hat{\epsilon}} \sqrt{\frac{g}{\epsilon \hat{\epsilon} \gamma}}-\frac{C}{\hat{\epsilon}}\left(\frac{a}{r}\right)^{1 / 8}\left[1+\frac{1}{\epsilon \xi(g / \gamma)} \frac{a}{r}\left(\frac{a}{r}\right)^{1 / 8}\right]\right) \\
& \cdot\left(1-C \epsilon-\frac{\epsilon}{2}\left(1+\frac{2}{N \epsilon^{2} \hat{\epsilon}}\right) \frac{g / \gamma}{E^{\mathrm{LL}}(1,1, g / \gamma)^{1+1 / s}}\right) .
\end{aligned}
$$

Here we used again (4.14) to estimate $R$ from above, and $\epsilon^{2} N \leq N_{\alpha} \leq N$ in the error term. This proves the lower bound in Region 3, as long as $a /(r \xi(g / \gamma))$ stays bounded (or at least does not increase too fast as $a / r$ goes to zero with $N$ ). Note that, for small $g / \gamma$,

$$
\frac{a}{r} \frac{1}{\xi(g / \gamma)} \sim \frac{a}{r}(\gamma / g)^{(s+2) /(s+1)} \sim \frac{r N}{\bar{L}_{\mathrm{TF}}},
$$

with $\bar{L}_{\mathrm{TF}}$ defined in (2.11), and hence the bound is uniform for $r N / \bar{L}_{\mathrm{TF}}$ bounded.

As explained briefly in the introduction to this section, if $N r / \bar{L}_{\mathrm{TF}}$ is not small, we have to use Thm. 3.2 instead of Thm. 3.1. Hence we will now assume that $A \equiv\left(r N / \bar{L}_{\mathrm{TF}}\right)^{1 / 3} \geq 1$, but still $g / \gamma \rightarrow 0$ as $N \rightarrow \infty$. Instead of (3.2) we will use the bound (3.4) in (4.22). This gives (4.22) with $\nu(n)$ replaced by

$$
\nu^{\prime \prime}(n)=C\left(\frac{n a}{\ell}+\chi^{1 / 14}+\left(\frac{n \ell a}{r^{2}}\right)^{1 / 8}+\left(\left[1+\frac{\sqrt{\chi} r}{\ell}\right]\left(\frac{\sqrt{n} a}{r}\right)^{1 / 4}\right]^{4 / 39}\right) .
$$

Let $0<\hat{\epsilon}<1$. For $N_{\alpha} \geq \max \left\{2 A^{2} / \hat{\epsilon}, \epsilon^{2} N\right\}$ we proceed as above, but choosing $\eta=A^{2} /\left(\delta N_{\alpha}\right)$ for $\frac{1}{2} \hat{\epsilon} \leq \delta \leq \hat{\epsilon}$. Moreover, we choose $\chi=1 /\left(\widetilde{\epsilon} N_{\alpha} \eta\right)$. Using $R \sim \bar{L}_{\mathrm{TF}}$, this gives (4.35), with $\mathcal{R}^{\prime}$ replaced by

$$
\begin{aligned}
\mathcal{R}^{\prime \prime}= & \left(1-C\left[\frac{N a}{\epsilon R}+\left(\frac{\hat{\epsilon}}{\widetilde{\epsilon} A^{2}}\right)^{\frac{1}{14}}+\left(\frac{a A}{r \epsilon \hat{\epsilon}^{2}}\right)^{\frac{1}{8}}+\left(\left[1+\frac{\hat{\epsilon}^{1 / 2}}{\epsilon \widetilde{\epsilon}^{1 / 2}}\right]\left(\frac{a A}{\hat{\epsilon} r}\right)^{1 / 4}\right)^{\frac{4}{39}}\right]\right) \\
& \cdot(1-\widetilde{\epsilon})^{3}\left(1-C \epsilon-\frac{\epsilon}{2}\left(1+\frac{2 A^{2}}{N \epsilon^{2} \hat{\epsilon}}\right) \frac{g / \gamma}{E^{\mathrm{LL}}(1,1, g / \gamma)^{1+1 / s}}\right) .
\end{aligned}
$$

Note that, for $g / \gamma \ll 1, N a / R \sim a \bar{\rho} \ll 1$, and $(a A / r)^{3}=(a / r)^{2} N a / R$. Moreover, $A^{2} / N \ll N^{-1 / 3}$ if $\bar{L}_{\mathrm{TF}} / L=(N g L)^{1 /(s+1)}$ is bounded away from zero. Hence, for bounded $1 / A$, this gives the desired lower bound for Region 3 .

In summary, we have thus shown that, in the limit $N \rightarrow \infty$ and $r / L \rightarrow 0$,

$$
\liminf \frac{E^{\mathrm{QM}}(N, L, r, a)-N e^{\perp} / r^{2}}{E^{\mathrm{LL}}(N, L, g)} \geq 1
$$

uniformly in the parameters, provided (1.12) holds, $a / r \rightarrow 0$ and $1 /(N g L)$ stays bounded. This finishes the proof of the lower bound for Regions 3-5. 


\subsection{Lower bound for Regions 1 and 2}

We are left with the lower bound for Regions 1 and 2. We proceed similarly to [26, Sect. 5.1]. Let $\rho_{N, L, g}^{\mathrm{GP}}$ denote the minimizer of the GP functional (2.4) under the normalization condition $\int_{\mathbb{R}} \rho=N$, and let $\phi(z)=\left(\rho_{N, L, g}^{\mathrm{GP}}(z)\right)^{1 / 2}$. We write a general wave function $\Psi$ as

$$
\Psi\left(\mathbf{x}_{1}, \ldots, \mathbf{x}_{N}\right)=F\left(\mathbf{x}_{1}, \ldots, \mathbf{x}_{N}\right) \prod_{k=1}^{N} \phi\left(z_{k}\right) b_{r}\left(\mathbf{x}_{k}^{\perp}\right)
$$

and assume that $\langle\Psi \mid \Psi\rangle=1$. In evaluating the expectation value of $H_{N, L, r, a}$, we use partial integration and the GP equation

$$
-\phi^{\prime \prime}+V \phi+g|\phi|^{2} \phi=\left(E^{\mathrm{GP}}(N, L, g)+\frac{g}{2} \int|\phi|^{4}\right) \phi .
$$

Moreover, we split a fraction of the kinetic energy into a part where the particles are closer than a distance $T>R_{0}$, and it's complement. This splitting will be important in the proof of BEC below. More precisely, for fixed $i$ and $\mathbf{x}_{j}, j \neq i$, let

$$
\chi_{i, T}(\mathbf{x})= \begin{cases}1 & \text { if } \min _{k, k \neq i}\left|\mathbf{x}-\mathbf{x}_{k}\right| \geq T \\ 0 & \text { otherwise }\end{cases}
$$

and let $\bar{\chi}_{i, T}=1-\chi_{i, T}$. Then, for $0 \leq \epsilon \leq 1$,

$$
\begin{aligned}
\left\langle\Psi\left|H_{N, L, r, a}\right| \Psi\right\rangle= & \frac{N e^{\perp}}{r^{2}}+E^{\mathrm{GP}}(N, L, g)+\frac{g}{2} \int|\phi|^{4}+Q(F) \\
& +(1-\epsilon) \int\left|\nabla_{i} F\right|^{2} \chi_{i, T}\left(\mathbf{x}_{i}\right) \prod_{k=1}^{N} \phi\left(z_{k}\right)^{2} b_{r}\left(\mathbf{x}_{k}^{\perp}\right)^{2} d^{3} \mathbf{x}_{k}
\end{aligned}
$$

with

$$
\begin{aligned}
Q(F)= & \int\left(\sum_{i=1}^{N}\left[\epsilon\left|\nabla_{i} F\right|^{2}+(1-\epsilon)\left|\nabla_{i} F\right|^{2} \bar{\chi}_{i, T}\left(\mathbf{x}_{i}\right)\right]\right. \\
& \left.+\sum_{i<j} v_{a}\left(\left|\mathbf{x}_{i}-\mathbf{x}_{j}\right|\right)|F|^{2}-g \sum_{i=1}^{N}\left|\phi\left(z_{i}\right)\right|^{2}|F|^{2}\right) \prod_{k=1}^{N} \phi\left(z_{k}\right)^{2} b_{r}\left(\mathbf{x}_{k}^{\perp}\right)^{2} d^{3} \mathbf{x}_{k} .
\end{aligned}
$$

To bound $Q(F)$ from below, we use again the box method. We divide $\mathbb{R}$ in $z$ direction into intervals of length $M$, labeled by $\alpha$, put $N_{\alpha}$ particles in box $\alpha$, neglect the interaction between boxes, and minimize over the distribution of the $N$ particles. This gives a lower bound. More precisely

$$
\inf _{F} Q(F) \geq \inf _{\left\{N_{\alpha}\right\}} \sum_{\alpha} \inf _{F_{\alpha}} Q_{\alpha}\left(F_{\alpha}\right)
$$


where $F_{\alpha}=F_{\alpha}\left(\mathbf{x}_{1}, \ldots, \mathbf{x}_{N_{\alpha}}\right)$, and $Q_{\alpha}$ is the same as $Q$, but with all the integrations restricted to the box $\alpha$, and $N$ replaced by $N_{\alpha}$. The infima are under the normalization conditions $\int|F|^{2} \prod_{k=1}^{N} \phi\left(z_{k}\right)^{2} b_{r}\left(\mathbf{x}_{k}^{\perp}\right)^{2}=1$ and $\int_{\alpha}\left|F_{\alpha}\right|^{2} \prod_{k=1}^{N_{\alpha}} \phi\left(z_{k}\right)^{2} b_{r}\left(\mathbf{x}_{k}^{\perp}\right)^{2}=1$, respectively.

We consider two cases. Choose $\delta>0$. First, assume that, for all $z \in \alpha,|\phi(z)|^{2} \geq$ $\delta N / L$. We use the same method as in [26, Eqs. (5.28)-(5.34)] to get rid of the $\phi^{2}$ in the measure $\phi(z)^{2} d z$. Let $\phi_{\alpha, \min }$ and $\phi_{\alpha, \max }$ denote the minimal and maximal value of $\phi$ inside the box $\alpha$, respectively. We first proceed as in (3.30)-(3.36), and obtain, for $T \geq r(a / r)^{1 / 4}=$ radius of $U$,

$$
\begin{aligned}
& \sum_{i=1}^{N_{\alpha}} \int_{\alpha}\left[(1-\epsilon)\left|\nabla_{i} F\right|^{2} \bar{\chi}_{i, T}\left(\mathbf{x}_{i}\right)+\frac{1}{2} \sum_{j, j \neq i} v_{a}\left(\left|\mathbf{x}_{i}-\mathbf{x}_{j}\right|\right)|F|^{2}\right] \prod_{k=1}^{N_{\alpha}} \phi\left(z_{k}\right)^{2} b_{r}\left(\mathbf{x}_{k}^{\perp}\right)^{2} d^{3} \mathbf{x}_{k} \\
& \geq \sum_{i=1}^{N_{\alpha}} \int_{\alpha}\left[\frac{\phi_{\alpha, \min }^{2}}{\phi_{\alpha, \max }^{2}} a^{\prime} U\left(\left|\mathbf{x}_{i}-\mathbf{x}_{k(i)}\right|\right) \chi_{\mathcal{B}_{\delta}}\left(\mathbf{x}_{k(i)}^{\perp} / r\right)|F|^{2}\right] \prod_{k=1}^{N_{\alpha}} \phi\left(z_{k}\right)^{2} b_{r}\left(\mathbf{x}_{k}^{\perp}\right)^{2} d^{3} \mathbf{x}_{k} .
\end{aligned}
$$

Here $U$ is given in (3.33), and $a^{\prime}$ is given after Eq. (3.36). Denoting

$$
\widetilde{F}\left(\mathbf{x}_{1}, \ldots, \mathbf{x}_{N_{\alpha}}\right)=F\left(\mathbf{x}_{1}, \ldots, \mathbf{x}_{N_{\alpha}}\right) \prod_{k=1}^{N_{\alpha}} \phi\left(z_{k}\right)
$$

and using

$$
\left|\nabla_{i} \widetilde{F}\right|^{2} \leq 2\left|\nabla_{i} F\right|^{2} \prod_{k=1}^{N_{\alpha}} \phi\left(z_{k}\right)^{2}+2|\widetilde{F}|^{2} \frac{\sup _{z \in \alpha}\left|\phi^{\prime}\right|^{2}}{\phi_{\alpha, \min }^{2}}
$$

we get

$$
\int_{\alpha}\left|\nabla_{i} F\right|^{2} \prod_{k=1}^{n} \phi\left(z_{k}\right)^{2} b_{r}\left(\mathbf{x}_{k}^{\perp}\right)^{2} d^{3} \mathbf{x}_{k} \geq \int_{\alpha}\left[\frac{1}{2}\left|\nabla_{i} \widetilde{F}\right|^{2}-\frac{C_{N g L}}{\delta L^{2}}|\widetilde{F}|^{2}\right] \prod_{k=1}^{N_{\alpha}} b_{r}\left(\mathbf{x}_{k}^{\perp}\right)^{2} d^{3} \mathbf{x}_{k}
$$

Here we denoted

$$
C_{N g L}=\frac{L^{3}}{N} \sup _{z}\left|\phi^{\prime}(z)\right|^{2}
$$

which, by scaling, depends only on $N g L$. Estimating $\phi^{2}$ by it's maximum in the last term in (4.45), we therefore have

$$
Q_{\alpha}(F) \geq \widetilde{Q}_{\alpha}(\widetilde{F})-N_{\alpha} g \phi_{\alpha, \max }^{2}-\epsilon \frac{C_{N g L}}{\delta L^{2}} N_{\alpha},
$$

with

$$
\widetilde{Q}_{\alpha}(F)=\sum_{i=1}^{N_{\alpha}} \int_{\alpha}\left(\frac{1}{2} \epsilon\left|\nabla_{i} F\right|^{2}+\frac{\phi_{\alpha, \min }^{2}}{\phi_{\alpha, \max }^{2}} a^{\prime} U\left(\left|\mathbf{x}_{i}-\mathbf{x}_{k(i)}\right|\right)|F|^{2}\right) \prod_{k=1}^{N} b_{r}\left(\mathbf{x}_{k}^{\perp}\right)^{2} d^{3} \mathbf{x}_{k} .
$$


Denote the infimum of $\widetilde{Q}_{\alpha}(F)$ over all $F$ (under the normalization condition $\left.\int|F|^{2} \prod_{k} b_{r}\left(\mathbf{x}_{k}^{\perp}\right)^{2} d^{3} \mathbf{x}_{k}=1\right)$ by $\widetilde{E}_{\alpha}\left(N_{\alpha}, M\right)$, and choose $\epsilon=(a / r)^{1 / 8}$. Looking at the proof of Thm. 3.1, we see that the lower bound in Subsect. 3.2 was obtained exactly from an expression like (4.53) (compare with (3.36)), except for the additional factor $\phi_{\alpha, \min }^{2} / \phi_{\alpha, \max }^{2}$. This factor can be estimated by

$$
\frac{\phi_{\alpha, \min }^{2}}{\phi_{\alpha, \max }^{2}} \geq 1-\frac{2 M}{L} \sqrt{\frac{C_{N g L}}{\delta}} .
$$

Therefore we can apply (3.2), and, in addition, Lemma 2 from Sunsection 3.4 to estimate $E_{\mathrm{N}}^{1 \mathrm{D}}$ from below. This gives

$$
\widetilde{E}_{\alpha}\left(N_{\alpha}, M\right) \geq \frac{1}{2} \frac{N_{\alpha}\left(N_{\alpha}-1\right)}{M} g\left(1-\frac{2 M}{L} \sqrt{\frac{C_{N g L}}{\delta}}\right)\left(1-C \nu\left(N_{\alpha}, M\right)\right),
$$

where

$$
\nu(N, M)=N \sqrt{g M}+N\left(\frac{a}{r}\right)^{1 / 8}\left[1+\frac{N r}{M}\left(\frac{a}{r}\right)^{1 / 8}\right] .
$$

Note that $\nu(N, M)$ is monotone increasing in $N$.

This bound is of no use for large $N_{\alpha}$, however. Therefore we will use again the box method, as in Subsect. 4.3 (see also [31]), with small boxes $\ell=M \eta$ for some $\eta^{-1} \in \mathbb{N}$. The use of Lemma 3 , with $L(x)=x[x-1]_{+}$and $\lambda=4$, implies

$$
\widetilde{E}_{\alpha}\left(N_{\alpha}, M\right) \geq \frac{1}{2} \frac{N_{\alpha}^{2}}{M} g\left(1-\frac{1}{N_{\alpha} \eta}\right)^{2}\left(1-\frac{2 M \eta}{L} \sqrt{\frac{C_{N g L}}{\delta}}\right)\left(1-C \nu\left(\left\langle 4 N_{\alpha} \eta\right\rangle, M \eta\right)\right) .
$$

Let $1 \geq \hat{\epsilon} \geq 2 / N$ such that $\hat{\epsilon} N \in \mathbb{N}$, and choose $\eta=(\hat{\epsilon} N)^{-1}$. We estimate $N_{\alpha} \leq N$ in the last term in (4.57), and choose $M=\epsilon L$ for some $\epsilon>\hat{\epsilon}$. Minimizing over $N_{\alpha}$ yields

$$
\widetilde{E}_{\alpha}\left(N_{\alpha}, M\right)-g \phi_{\alpha, \min }^{2} N_{\alpha} \geq-\frac{1}{2} g \phi_{\alpha, \min }^{4} M \mathcal{R},
$$

with

$$
\begin{aligned}
\mathcal{R}= & \left(1+\frac{\hat{\epsilon}}{2 \epsilon \delta}\right)^{2}\left(1-\frac{2 \epsilon}{\hat{\epsilon} N} \sqrt{\frac{C_{N g L}}{\delta}}\right)^{-1} \\
& \cdot\left(1-C\left[\sqrt{\frac{\epsilon g L}{\hat{\epsilon}^{3} N}}+\frac{1}{\hat{\epsilon}}\left(\frac{a}{r}\right)^{1 / 8}\left[1+\frac{r N}{\epsilon L}\left(\frac{a}{r}\right)^{1 / 8}\right]\right]\right)^{-1} .
\end{aligned}
$$

For boxes $\alpha$ where $\phi_{\alpha, \text { min }}^{2}<N \delta / L$, we just neglect the positive terms in (4.45). This gives

$$
\begin{aligned}
& E^{\mathrm{QM}}(N, L, r, a)-\left(1-\left(\frac{a}{r}\right)^{1 / 8}\right) \sum_{i=1}^{N} \int\left|\nabla_{i} F\right|^{2} \chi_{i, T}\left(\mathbf{x}_{i}\right) \prod_{k=1}^{N} \phi\left(z_{k}\right)^{2} b_{r}\left(\mathbf{x}_{k}^{\perp}\right)^{2} d^{3} \mathbf{x}_{k} \\
& \geq \frac{N e^{\perp}}{r^{2}}+E^{\mathrm{GP}}(N, L, g)+\frac{g}{2} \int|\phi|^{4}-\left(\frac{a}{r}\right)^{1 / 8} \frac{N}{L^{2}} \frac{C_{N g L}}{\delta} \\
& \quad+\sum_{\alpha} \inf _{N_{\alpha}}\left[\widetilde{E}\left(N_{\alpha}, M\right)-g \phi_{\alpha, \max }^{2} N_{\alpha}\right]-\delta \frac{N^{2} g}{L}
\end{aligned}
$$


Here we neglected the condition $\sum_{\alpha} N_{\alpha}=N$ on the $N_{\alpha}$, which can only lower the infimum. Moreover, the error in replacing $\phi_{\alpha, \max }^{2}$ by $\phi_{\alpha, \min }^{2}$ in the term in square brackets in (4.60) is bounded above by

$$
2 N g M \sup _{z} \phi(z)\left|\phi^{\prime}(z)\right| \equiv 2 N^{2} g \frac{M}{L^{2}} \hat{C}_{N g L} .
$$

Note that, by scaling, $\hat{C}_{N g L}$ depends only on $N g L$.

Using (4.58), $\sum_{\alpha} \phi_{\alpha, \min }^{4} M \leq \int \phi^{4}$ and $\sum_{\alpha} \phi_{\alpha, \min }^{2} M \leq N$ and dropping the positive second term on the left side of (4.60), we finally obtain

$$
\begin{aligned}
& E^{\mathrm{QM}}(N, L, r, a)-\frac{N e^{\perp}}{r^{2}}-E^{\mathrm{GP}}(N, L, g) \\
& \geq-\frac{g}{2} \int|\phi|^{4}(\mathcal{R}-1)-\left(\frac{a}{r}\right)^{1 / 8} \frac{N}{L^{2}} \frac{C_{N g L}}{\delta}-\delta N^{2} \frac{g}{L}-2 \epsilon N^{2} \frac{g}{L} \hat{C}_{N g L} .
\end{aligned}
$$

Note that $E^{\mathrm{GP}}(N, L, g) \geq \frac{1}{2} g \int \phi^{4}$ and $E^{\mathrm{GP}}(N, L, g) \geq c_{s} N / L^{2}$ for some constant $c_{s}$ depending only on $s$. Hence we see that, for bounded $N g L$, the parameters $\epsilon, \hat{\epsilon}$ and $\delta$ can be chosen arbitrary small with $a / r$ and $N$ to show the desired lower bound, as long as $N r / L \ll(r / a)^{1 / 4}$ and, in particular, for $N r / L$ bounded. (Note that $\bar{L} \sim L$ in Regions 1 and 2.) Here we also need that $C_{N g L}$ and $\hat{C}_{N g L}$ are uniformly bounded if $N g L$ stays bounded, which follows by the same methods as in the proof of Lemma 1 in the Appendix. For bigger values of $N r / L$ we have to proceed differently, using the lower bound (3.4) instead of (3.2), as we also did in the previous subsection for the lower bound for Region 3. We omit the details. The results of this subsection can thus be summarized as

$$
\liminf \frac{E^{\mathrm{QM}}(N, L, r, a)-N e^{\perp} / r^{2}}{E^{\mathrm{GP}}(N, L, g)} \geq 1
$$

in the limit $N \rightarrow \infty$ and $r / L \rightarrow 0$, uniformly in the parameters as long as $N g L$ stays bounded. This finishes the proof of the lower bound for Regions 1 and 2 .

\section{Bose-Einstein Condensation}

In this last section we investigate the question of Bose-Einstein condensation in the ground state. It will be proved to occur in Regions 1 and 2 but it probably also occurs in part of Region 3; we cannot prove this and it remains an open problem. BEC in the ground state means that the one-body density matrix $\gamma\left(\mathbf{x}, \mathbf{x}^{\prime}\right)$, which is obtained from the ground state wave function $\Psi_{0}$ by

$$
\gamma\left(\mathbf{x}, \mathbf{x}^{\prime}\right)=N \int \Psi_{0}\left(\mathbf{x}, \mathbf{x}_{2}, \ldots, \mathbf{x}_{N}\right) \Psi_{0}\left(\mathbf{x}^{\prime}, \mathbf{x}_{2}, \ldots, \mathbf{x}_{N}\right)^{*} d^{3} \mathbf{x}_{2} \cdots d^{3} \mathbf{x}_{N}
$$

factorizes as $N \psi(\mathbf{x}) \psi\left(\mathbf{x}^{\prime}\right)$ for some normalized $\psi$ (in the $N \rightarrow \infty$ limit, of course). This, in fact, is $100 \%$ condensation. It was proved in [25] for a fixed trap potential in the Gross-Pitaevskii limit, i.e., for both $r / L$ and $N a / L$ fixed as $N \rightarrow \infty$. Here we 
extend this result to the case $r / L \rightarrow 0$ with $N g L$ fixed. The function $\psi$ is the squareroot of the minimizer of the 1D GP functional (2.4) times the transverse function $b_{r}\left(\mathbf{x}^{\perp}\right)$.

BEC is not expected in Regions 4 and 5. Lenard [21] showed that the largest eigenvalue of $\gamma$ grows only as $N^{1 / 2}$ for a homogeneous gas of 1D impenetrable bosons and, according to [34] and [10], this holds also for a GT gas in a harmonic trap. (The exponent 0.59 in [14] can probably be ascribed to the small number of particles $(N=10)$ considered.)

Our main result about BEC in the ground state is:

Theorem 5.1 (BEC in Region 2). If $N \rightarrow \infty, r / L \rightarrow 0$ with $N a L / r^{2}$ fixed, then

$$
\frac{L r^{2}}{N} \gamma\left(r \mathbf{x}^{\perp}, L z ; r \mathbf{x}^{\prime \perp}, L z^{\prime}\right) \rightarrow b\left(\mathbf{x}^{\perp}\right) b\left(\mathbf{x}^{\prime \perp}\right) \phi^{\mathrm{GP}}(z) \phi^{\mathrm{GP}}\left(z^{\prime}\right)
$$

in trace norm. Here $\phi^{\mathrm{GP}}$ is the minimizer of the GP functional (2.4) with $N=1$, $L=1$ and interaction parameter $N g L$.

Proof. As in the proof of the energy asymptotics in Region 2, we have to distinguish the cases $r N / L$ small or large. For simplicity we consider only the case $r N / L \ll$ $(r / a)^{1 / 4}$. The case of larger $r N / L$ can be treated in the same manner, replacing the bound (3.2) by (3.4) in Subsect. 4.4, and choosing $T$ in (4.43) appropriately.

From the lower bound to the energy in Subsect. 4.4, together with the upper bound in Subsect. 4.1, we infer that if $\Psi_{0}$ is the ground state of the Hamiltonian $H_{N, L, r, a}, T=r(a / r)^{1 / 4}$ and $F$ is defined by

$$
\Psi_{0}\left(\mathbf{x}_{1}, \ldots, \mathbf{x}_{N}\right)=F\left(\mathbf{x}_{1}, \ldots, \mathbf{x}_{N}\right) \prod_{k=1}^{N} L^{-1 / 2} \phi^{\mathrm{GP}}\left(z_{k} / L\right) b_{r}\left(\mathbf{x}_{k}^{\perp}\right),
$$

then

$$
\lim _{N \rightarrow \infty} \frac{L^{2}}{N} \sum_{i=1}^{N} \int\left|\nabla_{i} F\right|^{2} \chi_{i, T}\left(\mathbf{x}_{i}\right) \prod_{k=1}^{N} L^{-1} \phi^{\mathrm{GP}}\left(z_{k} / L\right)^{2} b_{r}\left(\mathbf{x}_{k}^{\perp}\right)^{2} d^{3} \mathbf{x}_{k}=0
$$

in the limit $N \rightarrow \infty, r / L \rightarrow 0$ with $N g L$ fixed. Here $\chi_{i, T}$ is given in (4.43). Note that in this limit $N T^{3} /\left(r^{2} L\right)=N r / L(a / r)^{3 / 4} \rightarrow 0$, i.e., the volume of the set where $\chi_{i, T}$ is zero is small compared to the total volume $r^{2} L$. Eq. (5.2) now follows, using the methods of [25].

\section{A Appendix: Proof of auxiliary Lemmas}

Proof of Lemma 1. Without restriction we may assume that $V^{\perp} \geq 0$. The existence, uniqueness and positivity of a minimizer $\phi_{p}$ are standard (cf., e.g., [27]). From the variational equation

$$
\left(-\Delta^{\perp}+V^{\perp}\left(\mathbf{x}_{\perp}\right)+2 p\left|\phi_{p}\left(\mathbf{x}^{\perp}\right)\right|^{2}\right) \phi_{p}\left(\mathbf{x}^{\perp}\right)=\mu_{p} \phi_{p}\left(\mathbf{x}^{\perp}\right)
$$


we infer that, for $K\left(\mathbf{x}^{\perp}-\mathbf{y}^{\perp}\right)$ the integral kernel of $\left(-\Delta^{\perp}+1\right)^{-1}$,

$$
\phi_{p}\left(\mathbf{x}^{\perp}\right)=\int K\left(\mathbf{x}^{\perp}-\mathbf{y}^{\perp}\right)\left(\mu_{p}+1-V^{\perp}\left(\mathbf{y}^{\perp}\right)-2 p\left|\phi_{p}\left(\mathbf{y}^{\perp}\right)\right|^{2}\right) \phi_{p}\left(\mathbf{y}^{\perp}\right) d^{2} \mathbf{y}^{\perp} .
$$

Using positivity of $V^{\perp}, \phi_{p}$ and $K$, as well as the normalization of $\phi_{p}$, we obtain the bound

$$
\begin{aligned}
\phi_{p}\left(\mathbf{x}^{\perp}\right) e^{\left|\mathbf{x}^{\perp}\right|} & \leq \sup _{\mathbf{x}^{\perp}} \int_{V^{\perp}\left(\mathbf{y}^{\perp}\right) \leq \mu_{p}+1} e^{\left|\mathbf{x}^{\perp}\right|} K\left(\mathbf{x}^{\perp}-\mathbf{y}^{\perp}\right)\left(\mu_{p}+1\right) \phi_{p}\left(\mathbf{y}^{\perp}\right) d^{2} \mathbf{y}^{\perp} \\
& \leq\left(\mu_{p}+1\right) \sup _{\mathbf{x}^{\perp}}\left(\int_{V^{\perp}\left(\mathbf{y}^{\perp}\right) \leq \mu_{p}+1}\left|e^{\left|\mathbf{x}^{\perp}\right|} K\left(\mathbf{x}^{\perp}-\mathbf{y}^{\perp}\right)\right|^{2} d^{2} \mathbf{y}^{\perp}\right)^{1 / 2} \equiv C_{p} .
\end{aligned}
$$

Since $\mu_{p}$ is uniformly bounded for $p$ in a bounded interval, so is $C_{p}$. Moreover,

$$
\begin{aligned}
\left|\nabla^{\perp} \phi_{p}\left(\mathbf{x}^{\perp}\right)\right| & \leq\left.\int\left|\nabla^{\perp} K\left(\mathbf{x}^{\perp}-\mathbf{y}^{\perp}\right)\right|\left|\mu_{p}+1-V^{\perp}\left(\mathbf{y}^{\perp}\right)-2 p\right| \phi_{p}\left(\mathbf{y}^{\perp}\right)\right|^{2} \mid \phi_{p}\left(\mathbf{y}^{\perp}\right) d^{2} \mathbf{y}^{\perp} \\
& \leq \widetilde{C}_{p}\left\|\nabla^{\perp} K\right\|_{1}
\end{aligned}
$$

with

$$
\widetilde{C}_{p}=\left.\sup _{\mathbf{y}^{\perp}}\left|\mu_{p}+1-V^{\perp}\left(\mathbf{y}^{\perp}\right)-2 p\right| \phi_{p}\left(\mathbf{y}^{\perp}\right)\right|^{2} \mid \phi_{p}\left(\mathbf{y}^{\perp}\right),
$$

which is finite and uniformly bounded for bounded $p$ because of (A.3) and the fact that that $V^{\perp}$ is polynomially bounded at infinity by assumption.

Proof of Lemma 2. We write (1.3) as

$$
H_{n, g}=\sum_{j=1}^{n}-\frac{1}{2} \partial_{j}^{2}+\frac{1}{2} \sum_{i \neq j}\left[-\frac{1}{n-1} \partial_{j}^{2}+g \delta\left(z_{i}-z_{j}\right)\right] .
$$

To bound this expression from below, we want to use Temple's inequality, and for this purpose we have to smear out the $\delta$-function interaction. We use the following Lemma (compare with [2, Lemma 6.3]), whose proof can be found below.

Lemma 5. Let $\partial_{z}^{2}$ denote the Neumann Laplacian on an interval $[0, \ell]$, let $z_{0} \in$ $(0, \ell)$, and choose positive numbers $A, B$ and $\alpha$ such that $R \equiv B \arctan (B A / 2 \alpha) \leq$ $\min \left\{z_{0}, \ell-z_{0}\right\}$. Then

$$
-\alpha \partial_{z}^{2}+A \delta\left(z-z_{0}\right)-\frac{\alpha}{B^{2}} \theta\left(R-\left|z-z_{0}\right|\right) \geq 0 .
$$

We apply this result to the operator in square brackets in (A.6). This gives

$$
H_{n, g} \geq \sum_{j=1}^{n}-\frac{1}{2} \partial_{j}^{2}+\frac{1}{2} \sum_{i \neq j} \frac{1}{(n-1) B^{2}} \theta\left(R-\left|z_{j}-z_{i}\right|\right) \chi_{[R, \ell-R])}\left(z_{i}\right)
$$


with $R=B \arctan (B g(n-1) / 2)$ and $B>0$ arbitrary. Temple's inequality (2.61) implies that, for

$$
U\left(z_{1}, \ldots, z_{n}\right)=\frac{1}{2} \sum_{i \neq j} \frac{1}{(n-1) B^{2}} \theta\left(R-\left|z_{j}-z_{i}\right|\right) \chi_{[R, \ell-R])}\left(z_{i}\right)
$$

and $\left\langle U^{k}\right\rangle=\ell^{-n} \int U^{k} d z_{1} \ldots d z_{n}$

$$
E_{\mathrm{N}}^{1 \mathrm{D}}(n, \ell, g) \geq\langle U\rangle\left(1-\frac{\left\langle U^{2}\right\rangle}{\langle U\rangle} \frac{1}{\frac{1}{2} \pi^{2} / \ell^{2}-\langle U\rangle}\right),
$$

provided the term in the denominator is positive. By Schwarz' inequality,

$$
\left\langle U^{2}\right\rangle \leq \frac{n}{2 B^{2}}\langle U\rangle
$$

Moreover,

$$
\langle U\rangle=\frac{1}{2} \frac{n}{B^{2}} \frac{1}{\ell^{2}} \int_{0}^{\ell} d z \int_{R}^{\ell-R} d w \theta(R-|z-w|)=\frac{n}{B^{2}} \frac{R}{\ell^{2}}(\ell-2 R) .
$$

Using $x \geq \arctan (x) \geq x(1-x / 3)$ for $x \geq 0$, this leads to the estimate

$$
E_{\mathrm{N}}^{1 \mathrm{D}}(n, \ell, g) \geq \frac{1}{2} n(n-1) \frac{g}{\ell}\left(1-\frac{B^{2} g n}{\ell}-\frac{B g n}{6}\right)\left(1-\frac{n}{B^{2}\left(\pi^{2} / \ell^{2}-n(n-1) g / \ell\right)}\right),
$$

under the assumption $\pi^{2} / \ell^{2}>n(n-1) g / \ell$. Choosing $B=\ell\left(\ell g \pi^{2}\right)^{-1 / 4}$, this gives, for $\mathcal{R}=\left(n^{2} \ell g\right)^{1 / 2} / \pi$

$$
E_{\mathrm{N}}^{1 \mathrm{D}}(n, \ell, g) \geq \frac{1}{2} n(n-1) \frac{g}{\ell}\left(1-\mathcal{R}-\frac{\pi}{6 n^{1 / 2}} \mathcal{R}^{3 / 2}\right)\left(1-\frac{\mathcal{R}}{1-\mathcal{R}^{2}}\right)
$$

and proves the desired lower bound.

Proof of Lemma 5. Let $h$ denote the operator on the left side of (A.7). The function

$$
f(z)= \begin{cases}\cos \left(\left(R-\left|z-z_{0}\right|\right) / B\right) & \text { for } 0 \leq\left|z-z_{0}\right| \leq R \\ 1 & \text { otherwise }\end{cases}
$$

fulfills the Schrödinger equation $h f=0$, and since it is positive, it must be the ground state of $h$. Hence $h \geq 0$.

Proof of Lemma 3. Let $p=\langle\lambda N / M\rangle$. For $n \leq p$ we use the estimate

$$
E(n) \geq L(n) K(p),
$$

which follows from monotonicity of $K$. For $n \geq p$, we use superadditivity, which implies that

$$
E(n) \geq[n / p] E(p) \geq \frac{n}{2 p} L(p) K(p)
$$


where $[x]$ denotes the integer part of $x$. Now let $t=\sum_{n<p} c_{n} n$. By convexity of $L$ and the fact that $L(0)=0$,

$$
\sum_{n<p} c_{n} L(n) \geq M L(t / M)
$$

Hence

$$
\sum_{n \geq 0} c_{n} E(n) \geq K(p)\left[M L(t / M)+(N-t) \frac{L(p)}{2 p}\right] .
$$

To obtain a lower bound, we have to minimize the right side over all $0 \leq t \leq N$. Note that (A.19) is convex in $t$, hence the minimum is either taken at $t=N$, or, if it is taken at some $t_{0}<N$, it's right derivative at $t_{0}$ has to be positive. Using (3.83) this leads to

$$
\frac{L\left(\lambda t_{0} / M\right)}{2 \lambda t_{0} / M} \geq \frac{L(p)}{2 p} .
$$

By our assumptions on $L, L(x) / x$ is monotone increasing, and hence $t_{0} \geq p M / \lambda \geq N$. Thus we can set $t=N$ in (A.19), and obtain the desired lower bound.

\section{References}

[1] G.E. Astrakharchik and S. Giorgini, Quantum Monte Carlo study of the three- to one-dimensional crossover for a trapped Bose gas, Phys. Rev. A 66, 053614-1-6 (2002).

[2] B. Baumgartner, J.P. Solovej, and J. Yngvason, Atoms in Strong Magnetic Fields: The High Field Limit at Fixed Nuclear Charge, Commun. Math. Phys. 212, 703-724 (2000).

[3] D. Blume, Fermionization of a bosonic gas under highly elongated confinement: A diffusion quantum Monte Carlo study, Phys. Rev. A 66, 053613-1-8 (2002).

[4] K. Bongs, S. Burger, S. Dettmer, D. Hellweg, J. Artl, W. Ertmer, and K. Sengstok, Waveguides for Bose-Einstein condensates, Phys. Rev. A 63, 031602 (2001).

[5] E.A. Cornell and C.E. Wieman, Bose-Einstein condensation in a dilute gas, the first 70 years and some recent experiments, in: Les Prix Nobel 2001 (The Nobel Foundation, Stockholm, 2002), pp. 87-108. Reprinted in: Rev. Mod. Phys. 74, 875-893 (2002); Chem. Phys. Chem. 3, 476-493 (2002).

[6] K.K. Das, Highly anisotropic Bose-Einstein condensates: Crossover to lower dimensionality, Phys. Rev. A 66, 053612-1-7 (2002).

[7] K.K. Das, M.D. Girardeau, and E.M. Wright, Crossover from One to Three Dimensions for a Gas of Hard-Core Bosons, Phys. Rev. Lett. 89, 110402-1-4 (2002). 
[8] V. Dunjko, V. Lorent, and M. Olshanii, Bosons in Cigar-Shaped Traps: ThomasFermi Regime, Tonks-Girardeau Regime, and In Between, Phys. Rev. Lett. 86, 5413-5316 (2001).

[9] F.J. Dyson, Ground-State Energy of a Hard-Sphere Gas, Phys. Rev. 106, 20-26 (1957).

[10] P.J. Forrester, N.E. Frankel, T.M. Garoni, and N.S. Witte, Finite one dimensional impenetrable Bose systems: Occupation numbers, arXiv:cond-mat/ 0211126.

[11] D.M. Gangardt, G.V. Shlyapnikov, Local correlations in a strongly interacting $1 D$ Bose gas, New Journal of Physics 5 , 79, (2003)

[12] M. Girardeau, Relationship between Systems of Impenetrable Bosons and Fermions in One Dimension, J. Math. Phys. 1, 516-523 (1960).

[13] M.D. Girardeau and E.M. Wright, Bose-Fermi variational Theory for the BECTonks Crossover, Phys. Rev. Lett. 87, 210401-1-4 (2001).

[14] M.D. Girardeau, E.M. Wright, and J.M. Triscari, Ground-state properties of a one-dimensional system of hard-core bosons in a harmonic trap, Phys. Rev. A 63, 033601-1-6 (2001).

[15] A. Görlitz, J. M. Vogels, A.E. Leanhardt, C. Raman, T.L. Gustavson, J.R. AboShaeer, A.P. Chikkatur, S. Gupta, S. Inouye, T. Rosenband, and W. Ketterle, Realization of Bose-Einstein Condensates in Lower Dimension, Phys. Rev. Lett. 87, 130402-1-4 (2001).

[16] M. Greiner, I. Bloch, O. Mendel, T. Hänsch, and T. Esslinger, Exploring Phase Coherence in a 2D Lattice of Bose-Einstein Condensates, Phys. Rev. Lett. 87, 160405 (2001).

[17] A.D. Jackson and G.M. Kavoulakis, Lieb Mode in a Quasi-One-Dimensional Bose-Einstein Condensate of Atoms, Phys. Rev. Lett. 89, 070403 (2002).

[18] W. Ketterle, When atoms behave as waves: Bose-Einstein condensation and the atom laser, in: Les Prix Nobel 2001 (The Nobel Foundation, Stockholm, 2002), pp. 118-154. Reprinted in: Rev. Mod. Phys. 74, 1131-1151 (2002); Chem. Phys. Chem. 3, 736-753 (2002).

[19] E.B. Kolomeisky, T.J. Newman, J.P. Straley, and X. Qi, Low-Dimensional Bose Liquids: Beyond the Gross-Pitaevskii Approximation, Phys. Rev. Lett. 85, 1146-1149 (2000). R.K. Bhaduri and D. Sen, Comment on "Low-Dimensional Bose Liquids: Beyond the Gross-Pitaevskii Approximation", Phys. Rev. Lett. 86, 4708 (2001). Reply Phys. Rev. Lett. 86, 4709 (2001).

[20] S. Komineas and N. Papanicolaou, Vortex Rings and Lieb Modes in a Cylindrical Bose-Einstein Condensate, Phys. Rev. Lett. 89, 070402 (2002). 
[21] A. Lenard, Momentum distribution in the ground state of the one-dimensional system of impenetrable bosons, J. Math. Phys. 5, 930-943 (1964).

[22] E.H. Lieb and W. Liniger, Exact Analysis of an Interacting Bose Gas. I. The General Solution and the Ground State, Phys. Rev. 130, 1605-1616 (1963).

[23] E.H. Lieb, Exact Analysis of an Interacting Bose Gas. II. The Excitation Spectrum, Phys. Rev. 130, 1616-1624 (1963).

[24] E.H. Lieb and M. Loss, Analysis, second edition, American Mathematical Society (2001).

[25] E.H. Lieb and R. Seiringer, Proof of Bose-Einstein Condensation for Dilute Trapped Gases, Phys. Rev. Lett. 88, 170409-1-4 (2002).

[26] E.H. Lieb, R. Seiringer, J.P. Solovej, and J. Yngvason, The Ground State of the Bose Gas, in: Current Developments in Mathematics, 2001, 131-178, International Press, Cambridge (2002).

[27] E.H. Lieb, R. Seiringer, and J. Yngvason, Bosons in a trap: A rigorous derivation of the Gross-Pitaevskii energy functional, Phys. Rev. A 61, 043602-1-13 (2000).

[28] E.H. Lieb, R. Seiringer, and J. Yngvason, A Rigorous Derivation of the GrossPitaevskii Energy Functional for a Two-dimensional Bose Gas, Commun. Math. Phys. 224, 17-31 (2001).

[29] E.H. Lieb, R. Seiringer, and J. Yngvason, One-dimensional Bosons in Threedimensional Traps, arXiv:cond-mat/0304071.

[30] E.H. Lieb, J.P. Solovej, and J. Yngvason, Asymptotics of Heavy Atoms in High Magnetic Fields. I : Lowest Landau Band Regions, Commun. Pure and Appl. Math. 47, 513-593 (1994).

[31] E.H. Lieb and J. Yngvason, Ground State Energy of the Low Density Bose Gas, Phys. Rev. Lett. 80, 2504-2507 (1998).

[32] C. Menotti, S. Stringari, Collective Oscillations of a 1D Trapped Bose gas, Phys. Rev. A 66, 043610 (2002)

[33] M. Olshanii, Atomic Scattering in the Presence of an External Confinement and a Gas of Impenetrable Bosons, Phys. Rev. Lett. 81, 938-941 (1998).

[34] T. Papenbrock, Ground-state properties of hard-core bosons in one-dimensional harmonic traps, Phys. Rev. A 67, 041601(R) (2003).

[35] D.S. Petrov, G.V. Shlyapnikov, and J.T.M. Walraven, Regimes of Quantum Degeneracy in Trapped 1 D Gases, Phys. Rev. Lett. 85, 3745-3749 (2000). 
[36] L. Pitaevskii and S. Stringari, Uncertainty Principle, Quantum Fluctuations, and Broken Symmetries, J. Low Temp. Phys. 85, 377-388 (1991).

[37] D.W. Robinson, The Thermodynamic Pressure in Quantum Statistical Mechanics, Lecture Notes in Physics, Vol. 9, Springer (1971).

[38] F. Schreck, L. Khaykovich, K.L. Corwin, G. Ferrari, T. Boudriel, J. Cubizolles, and C. Salomon, Quasipure Bose-Einstein Condensate Immersed in a Fermi Sea, Phys. Rev. Lett. 87, 080403 (2001).

[39] B. Tanatar and K. Erkan, Strongly interacting one-dimensional Bose-Einstein condensates in harmonic traps, Phys. Rev. A 62, 053601-1-6 (2000). M.D. Girardeau and E.M. Wright, Comment on "Strongly interacting one-dimensional Bose-Einstein condensates in harmonic traps", arXiv:cond-mat/0010457.

[40] G. Temple, The theory of Rayleigh's Principle as Applied to Continuous Systems, Proc. Roy. Soc. London A 119, 276-293 (1928).

[41] L. Tonks, The Complete Equation of State of One, Two and Three-Dimensional Gases of Hard Elastic Spheres, Phys. Rev. 50, 955-963 (1936). 